Spring 6-8-2021

\title{
Qualitative Exploration of Mealtime Routines and Parental Stress of Children with Pediatric Feeding Disorders: Analysis of Occupational Performance
}

\author{
Andrea Patino \\ University of St. Augustine for Health Sciences \\ DOI: https://doi.org/10.46409/sr.EQNP2360
}

Follow this and additional works at: https://soar.usa.edu/capstones

Part of the Occupational Therapy Commons, and the Pediatrics Commons

\section{Recommended Citation}

Patino, A. (2021). Qualitative Exploration of Mealtime Routines and Parental Stress of Children with Pediatric Feeding Disorders: Analysis of Occupational Performance. [Doctoral project, University of St Augustine for Health Sciences]. SOAR @ USA: Student Capstone Projects Collection. https://doi.org/ $10.46409 /$ sr.EQNP2360

This Capstone is brought to you for free and open access by the Student Research at SOAR @ USA. It has been accepted for inclusion in Student Capstone Projects by an authorized administrator of SOAR @ USA. For more information, please contact soar@usa.edu, erobinson@usa.edu. 
Spring 6-8-2021

Qualitative Exploration of Mealtime Routines and Parental Stress of Children with Pediatric Feeding Disorders: Analysis of Occupational Performance

Andrea Patino

Follow this and additional works at: https://soar.usa.edu/capstones

Part of the Occupational Therapy Commons 
Qualitative Exploration of Mealtime Routines and Parental Stress of Children with Pediatric Feeding Disorders: Analysis of Occupational Performance

Andrea Patino

Department of Occupational Therapy, University of St. Augustine for Health Sciences

A Capstone Presented in Partial Fulfillment of the Requirement for the Degree of DOCTOR OF OCCUPATIONAL THERAPY University of St. Augustine for Health Sciences April, 2021 
Qualitative Exploration of Mealtime Routines and Parental Stress of Children with Pediatric Feeding Disorders: Analysis of Occupational Performance

Andrea Patino

Department of Occupational Therapy, University of St. Augustine for Health Sciences has been approved

April, 2021

APPROVED:

Pamela Kasyan-Howe, OTD, OTR/L, Doctoral Coordinator

Kristin Domville, Dr. OT, OTR/L, Doctoral Coordinator

Jose Rafols, OTD, MHSA, OTR/L, OTR/L, BCTS, CEAS, Program Director

ACCEPTED AND SIGNED:

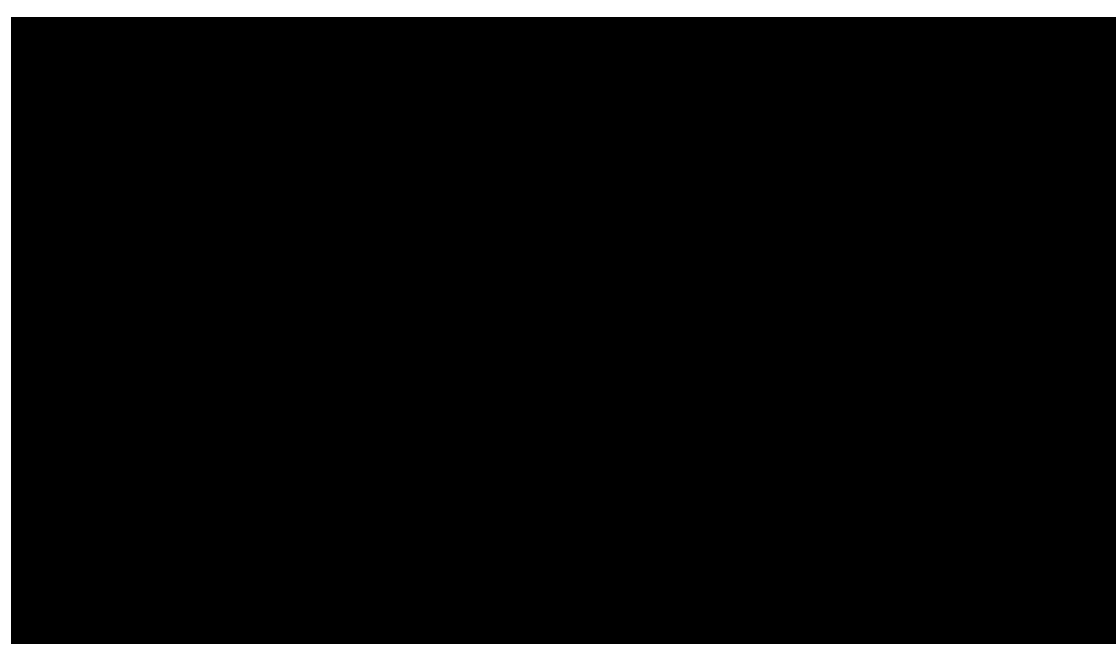




\section{Table of Contents}

Chapter I: Introduction............................................................

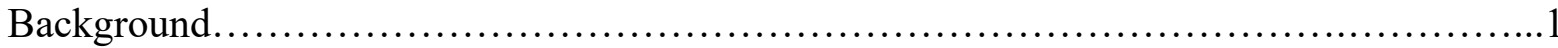

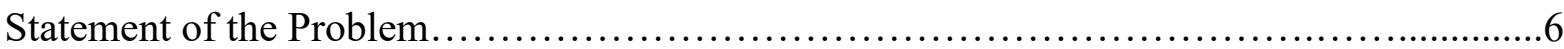

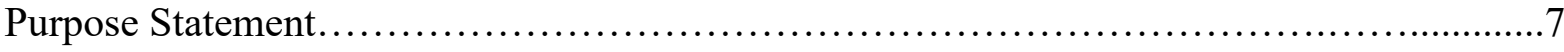

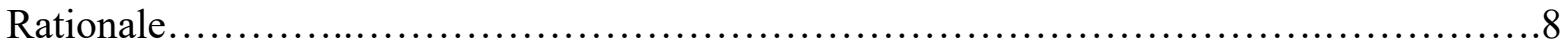

Significance............................................................. 9

Objectives...................................................................

Learning Objectives........................................................

Project Objectives........................................................ 10

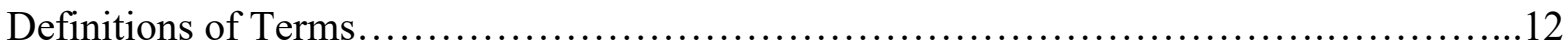

Assumptions............................................................... 12

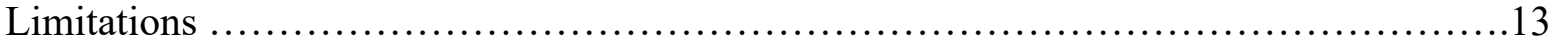

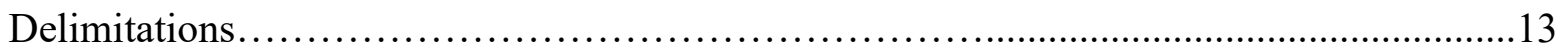

Chapter II: Literature Review................................................14

Pediatric Feeding Disorders ................................................... 15

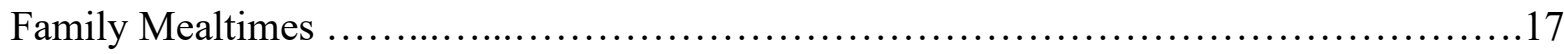

Parenting During Mealtimes...........................................................

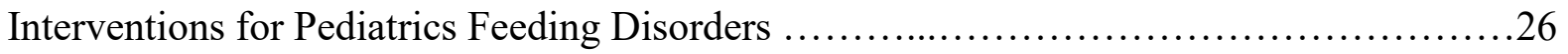

Occupational Therapy and Pediatric Feeding Disorders..............................27

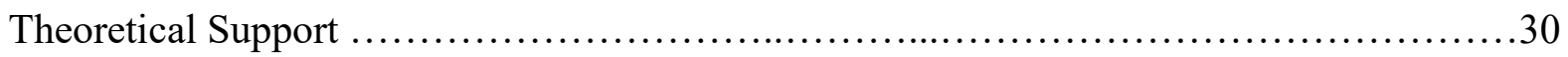

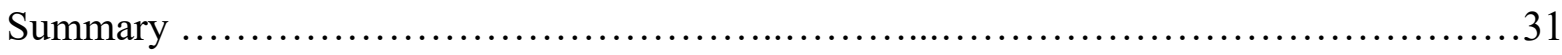

Chapter III: Methodology........................................................ 33 


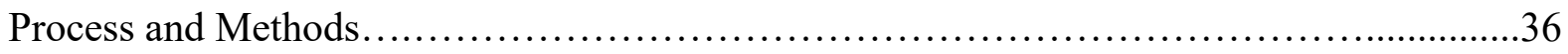

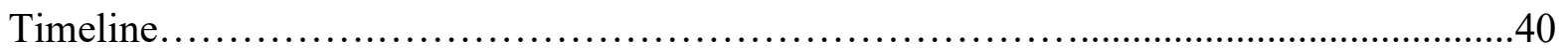

Conclusion and Next Steps....................................................... 41

Chapter IV: Results and Analysis.....................................................42

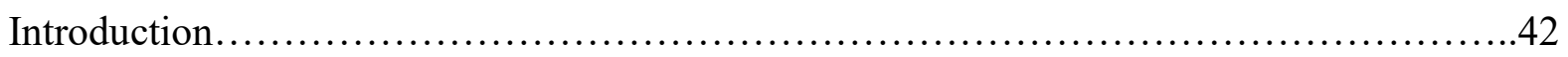

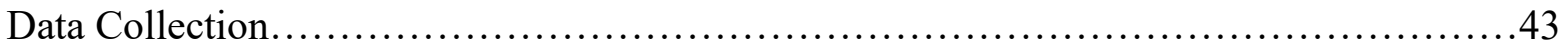

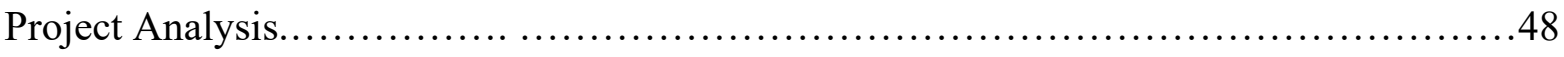

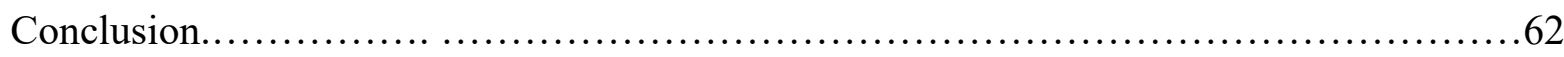

Chapter V: Summary, Discussion, and Conclusion.......................................64

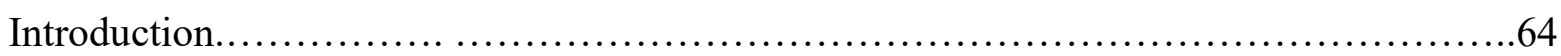

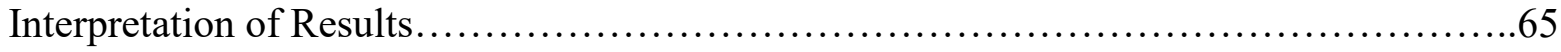

Strengths and Limitations.......................................................... 71

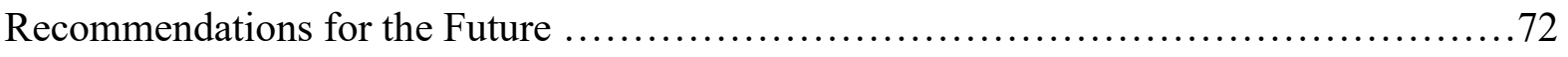

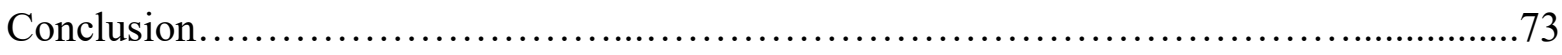

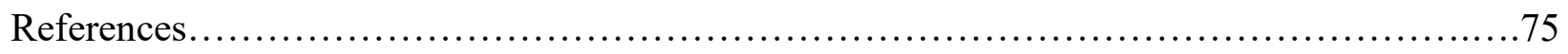

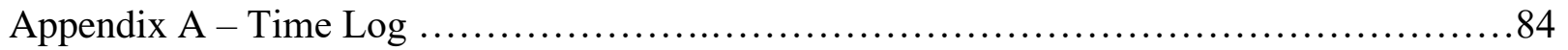

Appendix B- Participant Information Sheet............................................... 89

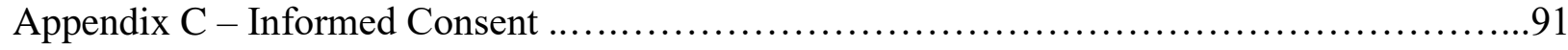

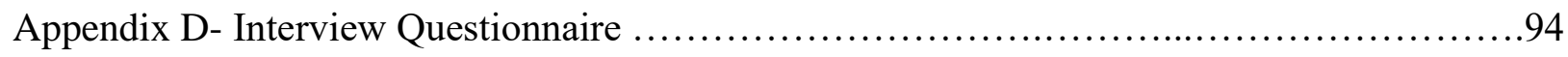

Appendix E- Memorandum of Understanding .......................................... 98 
Copyright (C) Andrea Patino, 2021 all rights reserved 


\section{Qualitative Exploration of Mealtime Routines and Parental Stress of Children with Pediatric Feeding Disorders: Analysis of Occupational Performance}

\section{Chapter 1: Introduction}

The purpose of this chapter is to provide background information about pediatric feeding disorders and how they affect mealtime routines, mealtime behaviors, and parental stress. This chapter will also have information on current occupational therapy interventions and the models that are guiding the project.

\section{Background}

Occupational therapy focuses on client-centered care that facilitates independence in meaningful occupations, including feeding and eating. According to the American Occupational Therapy Association (AOTA, 2017), the areas of occupation of feeding and eating are essential and complex tasks required for human functioning, which involve multiple systems such as, nervous, digestive, respiratory, muscular, endocrine. The complexity of these tasks requires a coordinated interaction between all systems to engage in each stage of the feeding and eating process (Goday et al., 2019). Children are at risk of developing a feeding disorder if they experience a disruption in any of the systems (Goday et al., 2019). 25-35\% of children with normal intellectual development are diagnosed with a Pediatric Feeding Disorder or PFD (Yang, 2017). For children with developmental disabilities, the number increases to $40-80 \%$ and $90 \%$ for children with autism spectrum disorders, or ASD (autism spectrum disorder) (Yang, 2017). Pediatric Feeding Disorders and Autism Spectrum Disorders have a significant impact on the child's development and growth. As a direct consequence of feeding disorders, children's physical, emotional, and cognitive functions are impacted causing developmental impairments, failure-to-thrive, and poor growth (Estrem et al., 2018; Gonzalez \& Stern, 2016). 
Pediatric feeding disorders affect families by disrupting mealtime routines, changing family dynamics, and increasing parental stress (Ausderau et al., 2019; Crowe et al., 2016; Johnson et al., 2019; Postorino et al., 2015). Mealtime routines are defined as the schedule of meals or snacks, exposure to food groups, presentation, setting, and family members involved (Bahr \& Johanson, 2013). According to Gedney (2013), family mealtimes are an essential component of the overall family dynamic. Mealtimes provide a sense of achievement and an overall increase in family quality of life (Crowe et al., 2016; Curtiss \& Ebata, 2018; Fishbein et al., 2016). For families to have a positive mealtime experience, the mealtime routines must be consistent, relaxed, and free of negative feeding practices such as forceful feeding and positively reinforcing negative behaviors. Marshall et al. (2014) found 57\% of studies, reported that parents expressed concern over their child's mealtime behaviors, reporting that behaviors affected their routines, lifestyle and increased stress. Furthermore, Curtin et al. (2015) found that little research has been conducted to establish a relationship between PFD and mealtime behaviors, which can affect parental stress and influence changes in the family dynamic.

Impacts on development include delays or changes in gross and fine motor coordination, food texture transitions, and oral motor functions (Goday et al., 2019). To achieve appropriate development, children must acquire the necessary skills and mechanisms to engage in the occupation of feeding successfully. External variables such as family dynamics, environment, and feedback during meals are essential for the reciprocal relationship between child and parent (Estrem et al., 2018). The first interactions a child has with their mother are revolved around feeding, and it is the foundation for bonding and attachment (Bahr \& Johanson, 2013). Family mealtime routines offer children an opportunity to develop social-emotional and cognitive abilities (Bahr \& Johanson, 2013). The process of feeding is essential for the child's growth and 
development and has many benefits including better health, nutritional status, and positive family interactions (Bahr \& Johanson, 2013; Gonzalez \& Stern, 2016). Mealtimes are an excellent way for parents to create routines that will positively benefit their child's growth and development.

Mealtimes are unique for every family, with some encouraging a more structured mealtime routine, while others are more flexible with their approach (Ausderau \& Juarez, 2013; Ausderau et al., 2019; Fiese, Foley, \& Spagnola, 2006). In many cases, children with feeding disorders require separate meals and mealtimes, in turn, affecting the way family members interact with one another and decreasing the opportunity for family engagement (Ausderau et al., 2019; Ausderau \& Juarez, 2013). Often, children with PFD and co-occurring ASD display maladaptive mealtime behaviors such as tantrums, aggression, head-turning, throwing, and spitting food. These behaviors affect mealtime routines, family dynamics, and parental stress (Johnson et al., 2019). Because many variables influence behaviors, it is essential to gather information from the family about the strategies utilized to manage the behaviors and parental stress.

Parental stress can be defined as increased emotional anxiety and changes in mood during mealtime routines (Didehbani et al., 2011; Crowe et al., 2016; Silverman et al., 2020). Elevated levels of stress during mealtimes affects the child-parent relationship and can lead the parent to adapt and cope or to continue reinforcing the child's feeding problems by implementing negative responses (Didehbani, 2009; Gonzalez \& Stern, 2016). Parents spend increased time providing physical assistance, prompting, monitoring, and encouraging the child during mealtimes, which has shown to contribute to an increase in overall parental stress (Ausderau \& Juarez, 2013; Crowe et al., 2016; Marshall et al., 2015). Some practices that reinforce feeding problems include forceful feeding and allowing the child to run away when presented with non-preferred 
foods (Didehbani, 2009). Didehbani (2009) found that specific parent intervention and training on stress reduction strategies may increase the parents' ability to respond to the child's particular needs during mealtime. Parents require assistance from healthcare professionals to develop the techniques that best work for the child and family.

Children with feeding disorders and co-occurring ASD often receive therapy (i.e., occupational therapy (OT), physical therapy (PT), speech-language pathologist (SLP), and feeding therapy. Although the children receive these services, parents are not receiving the adequate support to carry out the mealtime strategies learned at the clinic in the home environment (Johnson et al., 2015). In many cases, due to the elevated level of stress, parents cannot implement specific intervention outside the clinic setting to benefit their child (Thullen \& Bonsall, 2017). Didehbani et al. (2011) found that the implementation of parent training into the program positively impacted the child and parent's progress and a decrease in the child's negative mealtime behaviors. Thus, incorporating parents in clinic-based interventions and implementing parent training is essential to maximize the effects of therapy in the home environment and reduce the occurrence of behaviors during mealtimes.

Feeding disorders are complex and treated utilizing a specialized multidisciplinary feeding-team approach (Yang, 2017). The team may include physicians, nutritionists, psychologists, behavioral therapists, speech-language pathologists, and occupational therapists (Yang, 2017). Each profession provides expertise that will lead the child to optimal development. The nutritionist focuses on identifying meal plans suitable for the child's development based on their diagnosis (Didehbani, 2009). Behavioral therapists focus on positive reinforcement, stimulus fading, and escape extension (Sharp et al., 2017). Speech therapists emphasize the importance of oral motor functions and skills, as well as food textures that enable the child to 
engage in eating (Didehbani, 2009). While these professions offer excellent treatment strategies, occupational therapists consider all aspects of mealtime routine including family routines, rituals, and mealtime environment.

Occupational therapists focus on the fine and gross motor skills required for feeding, positioning, environmental modifications, and adaptive equipment required for proper feeding (Paul \& D’Amico, 2013). While also assisting, training, and educating parents on effective mealtime strategies that can be implemented at home. Occupational therapists use an individualized approach that addresses all components of the feeding program and tailor interventions to the family's needs (Ausderau et al., 2019). Another part of the occupational therapy intervention plan is to provide a holistic approach and acknowledge the importance of how mealtime routines dictate mealtime goals by addressing the barriers to optimal performance. It is within occupational therapy's scope of practice to investigate and determine interventions and strategies that focus on improving the multiple components that surround mealtime participation (Ausderau et al., 2019).

Occupational therapy practitioners utilize theoretical models to support and guide specific interventions. The theoretical models that support and align with the proposed project are the Ecology of Human Performance (EHP) and Person-Environment-Occupation-Performance (PEOP). EHP focuses on how the family interacts with the environment during mealtime routines while encompassing social and cultural customs and beliefs (Cole \& Tufano, 2008). The PEOP Model includes concepts such as adaptation fulfillment, mastery, and self-identify which will guide the development of the qualitative research questionnaire that will be used to interview the parents of children with PFD (Cole \& Tufano, 2008). PEOP also integrates neurobehavioral, cognitive, psychological, and spiritual factors that are all key components to 
addressing maladaptive behaviors and parental stress during mealtimes. These models will help guide the parent-directed interviews because it is essential to encompass all aspects of mealtime routines including social, cultural, and environmental factors.

\section{Statement of the Problem}

The problem is that few research studies show how parents manage behaviors and stress during mealtime routines (Ausderau et al., 2019; Crowe et al., 2016; Johnson et al., 2019; Postorino et al., 2015). Families of children diagnosed with PFD experience changes in mealtime routines, family dynamics, and parental stress. Feeding disorders affect the child's health, development, and interaction with family members, yet little attention has been placed on how PFD can affect parental stress. Because of feeding disorders on the child and families, children frequently display maladaptive behaviors during mealtime, which increases the overall parental stress. Fishbein et al. (2016) found that although three-fourths of the referral population were receiving services, parents sought additional services to assist with coping with stressors associated with feeding disorders.

Current OT services for PFD focus on oral intake, meal interventions, mealtime behaviors, and caregiver support and training (Gonzalez \& Stern, 2016). Even with these services, there is a low level of specificity regarding parent training, and parents have difficulty carrying over techniques learned in the clinic into other settings (Thullen \& Bonsall, 2017). It is within occupational therapy's scope of practice to focus on supporting these families by training and educating. Learning how parents manage stress and mealtime behaviors is essential for occupational therapists because this knowledge assists in creating new strategies that can be incorporated during evidence-based parent interventions. Occupational therapists have the expertise to treat the aspects of feeding that pertain to mealtime behaviors and parental stress. 


\section{Purpose Statement}

The purpose of this qualitative research type capstone project is to identify and analyze how parents of children with PFD manage maladaptive mealtime behaviors and parental stress. Parents of children undergoing feeding therapy and/or other services for PFD oversee the carryover of techniques to promote feeding and stability from the clinic setting into the home environment (Sharp et al., 2017; Silverman et al., 2020). The carry-over of these techniques is affected by the child's behavior and parental stress during mealtimes.

Information about strategies used to manage mealtime behaviors and stress will be collected during a face-to-face interview with the parents. The researcher will derive themes from the parent responses to identify strategies used to manage mealtime behaviors and parental stress. The data gathered from the interview and literature review will inform occupational therapists how to better support and train parents in improving and creating positive engagement in mealtime participation. This can be accomplished by incorporating parental strategies with evidence-based interventions to decrease maladaptive behaviors and parental stress.

The proposed project will look to answer the following research questions: (1) how do parents of children with PFD perceive maladaptive behaviors during mealtime routines? (2) how do parents of children with PFD describe strategies to manage maladaptive behaviors during mealtime? And (3) how do parents of children with PFD view their roles when managing stressors during mealtime? The student investigator hypothesized that parents perceive mealtime as more challenging due to the presence of maladaptive mealtime behaviors. It is also hypothesized that parents will describe managing behaviors in form of negative or positive reinforcement, punishment, or rewards. Lastly, it is hypothesized that parents view their daily roles as overwhelming when dealing with stress during mealtimes. 


\section{Rationale}

The proposed qualitative research project is essential to the profession of occupational therapy because it will help guide OT family-based interventions reinforcing the importance of carry-over strategies for parents. The proposed project reinforces the value of occupational therapy services that focus on family-based interventions and family-based training taking into consideration rituals, routines, and environment to help decrease parental stress during mealtime routines. The research findings will benefit the profession of occupational therapy because it will improve the quality of care and interventions provided to this population.

The proposed project will benefit the population of families of children with PFD because it will provide additional resources and advocacy for parents. The benefit of having more resources available for parents regarding mealtime stress and mealtime behaviors is that parents will have more opportunities to find a strategy that will benefit the family routine and dynamic. As mentioned previously, few research studies have investigated how parents manage behaviors and stress during mealtime. This study will allow parents the opportunity to answer questions and provide feedback on how occupational therapy can further assists during mealtimes.

Overall, the proposed project will hope to improve family interactions during mealtime routines and identify successful interventions strategies to decrease parental stress. The pursuit of this project is important to the profession of OT, parents, and children because it provides increased knowledge on how to provide family-centered care that addresses both maladaptive behaviors and parental stress during mealtime routines with children who have PFD. 


\section{Significance}

The significance of this research study is to fill the gap in knowledge regarding how parents manage maladaptive mealtime behaviors and mealtime stress to improve their occupational role of child rearing. The research study lies within the field of occupational therapy because it is client-centered, rooted in occupation, and considers the diversity of each client and family. The proposed project will assist occupational therapists in identifying themes across families on strategies used to decrease the occurrence of maladaptive behaviors that affect mealtime routines, family dynamics, and parental stress. Thullen \& Bonsall (2017) described that future research should focus on a holistic intervention that focuses on how parents manage stress and disruptive behaviors during mealtime routines. Another study by Curtin et al. (2015) found that little research has been done to establish a relationship between feeding disorders and mealtime behaviors, which can affect parental stress and influence changes in the family dynamic. The study is a step closer to identifying parents' needs during mealtime and providing client-centered holistic interventions and strategies for parents to utilize in the home environment.

\section{Objectives}

The proposed project will be supported by the following objectives:

\section{Learning Objectives:}

- Compare and contrast the current multidisciplinary interventions being implemented for children with feeding disorders

- This objective will be measured by creating a reflection paper demonstrating the multidisciplinary interventions being implemented at a hospital in a southern state.

- Compare and contrast the literature to parental responses from interview questionnaire 
○ This objective will be measured by creating a reflection paper outlining the differences and similarities between literature and parental responses.

- Develop broader understanding of the application of interventions in the home environment

○ This objective will be measured by asking parents during weekly meetings how they implement different strategies learned in the clinic in the home environment. The findings will be reflected in a reflection paper.

- Evaluate the family characteristics (socioeconomic status, child's gender, number of children) that may impact frequency of the intervention implementation

○ This objective will be measured by gathering sociodemographic information during the interview questionnaire. The data gathered will be put into a chart and any other information will be available in the reflection paper.

- Describe advantages and disadvantages of current strategies used by parents to manage maladaptive behaviors and parental stress

○ This information will be gathered during parent interviews and the data will be available in chapter four.

- Generalize themes from interviews and questionnaires that will help guide future interventions

- Themes will be derived, and information will be reflected in chapter four.

\section{Project Objectives:}

- Introduce project to parents of children in the intensive feeding program

- This objective will be accomplished by handing out a participant information sheet at a hospital in a southern state.

- Interview parents of children with PFD 
○ This objective will be accomplished by completing in-person interviews.

- Transcribing interview data

- The student investigator will transcribe data verbatim

- Input data into Dedoose.

- Analyze and report stressors parents experience during mealtime routines

○ This objective will be measured by gathering data from parent interviews and analyzing the most frequently occurring stressors during mealtime routines.

- Analyze and report reduction techniques to improve parent-child interactions

○ This objective will be measured by gathering data from the parent interviews and analyzing the most frequently used stress reduction strategies and how they impact parent-child relationships.

- Analyze and report most frequently occurring maladaptive behaviors and the strategies used by parents to manage these behaviors

- This objective will be measured by gathering data from the parent interviews and analyzing the most frequently occurring maladaptive mealtime behaviors.

- Analyze and report the similarities and differences of strategies used by families

$\circ$ This objective will be measured by gathering data from the parent interviews and analyzing the similarities and differences between parental responses.

- Attending weekly meetings with site mentor

- Share study Findings with facility

- Create a poster for dissemination using USAHS (University of Saint Augustine for Health Sciences) template

- Write Chapters 4 \& 5 of the Capstone paper 
- Submit completed and approved project

\section{Definition of Terms}

- Family dynamics: "are the patterns of relating, or interactions, between family members.” (Strong bonds, 2009, p.1).

- Family characteristics: Culture, Marital status, Co-parenting style, socio-economic status, occupation, and number of children in the family (Strong bonds, 2009, p.1).

- Child characteristics: age, gender, type of feeding problem, type of co-occurring medical conditions (Strong bonds, 2009, p.1).

- Parent characteristics: age, educational background, occupation, annual income, parental style, role, and skills (Murphy et al., 2019)

- Mealtime routines: schedule of meals/snacks, exposure to food groups, presentation, setting, and family members involved (Bahr \& Johanson, 2013)

- Feeding disorders: "impaired oral intake that is not age-appropriate and is associated with medical, nutritional, feeding skill, and/or psychosocial dysfunction" (Goday et al., 2019).

- Maladaptive behaviors: tantrums, aggression, head-turning, throwing, and spitting food, food refusal, and noncompliance (Aviram et al., 2020)

\section{Assumptions}

An assumption that must be considered in this research study is that the parents participating in the study will answer the interview questions truthfully. To minimize this possibility, the informed consent will state that the interviews will be anonymous and any information that may be used to identify the participant will not be published. Even though the participants may withdraw from the study at any time, it is assumed that they will complete the 
survey entirely. By utilizing a qualitative research method, it is assumed that the most reliable data will be collected to prove the hypotheses to be true. Another important assumption is that the sample is representative of the population overall.

\section{Limitations}

Current limitations for the proposed study include the availability of participants for the study. To minimize this possibility, the inclusion criteria will be any parent or primary caregiver of a child who currently has a pediatric feeding disorder diagnosis. Parents will not be excluded on whether their child is undergoing intensive feeding therapy involving an interdisciplinary team or outpatient feeding therapy. Another limitation to the study is the ongoing COVID-19 pandemic which may limit access to participants due to hospitals/clinics not allowing parents in the waiting rooms.

\section{Delimitations}

This study will only include parents of children with a pediatric feeding disorder diagnosis. The participants will only be recruited from a hospital in a southern state. The interview questionnaire is only to be completed in person and the audio must be recorded to ensure that the information transcribed is accurate. The interview questions will only be about mealtime routines, mealtime behavior, and parental stress. The proposed project will only focus on answering the following questions: (1) how do parents of children with PFD perceive maladaptive behaviors during mealtime? (2) how do parents of children with PFD describe strategies to manage maladaptive mealtime behaviors during mealtime? And (3) how do parents of children with PFD view their roles when managing stressors during mealtime? 


\section{Chapter II: Literature Review}

The purpose of this literature review is to provide an overview of already existing literature regarding how parents of children with pediatric feeding disorders manage maladaptive behaviors and parental stress during mealtime routines. The problem is that few research studies show how parents manage behaviors and stress during mealtime routines. This chapter will further explore the common themes presented when a thorough review of the literature was conducted. A review was conducted using PubMed and ProQuest databases while using the following key terms: pediatric feeding disorders, occupational therapy, feeding problems, parental stress, mealtime routines, mealtime behaviors. There will be an emphasis on comparing what the literature says about pediatric feeding disorders, family mealtimes, parenting during mealtimes, and interventions for pediatric feeding disorders.

The purpose of this qualitative research type capstone project is to identify and analyze how parents of children with PFD manage mealtime behaviors and parental stress. This will be accomplished through three primary purposes. The first will be to review the literature to identify and analyze the factors that affect mealtime routines and parental stress. This information will be used to guide interviews with parents of children diagnosed with PFD. Following the interview, the student investigator will derive themes from the parent responses to determine aspects of their mealtime routines that trigger stress and maladaptive behaviors and the coping strategies utilized to manage. Finally, the data gathered from the literature and interviews will inform occupational therapists how to better support parents in improving and creating a positive engagement in mealtime participation. This can be accomplished by analyzing, modifying, and adapting, as necessary. 
The following themes emerged from the literature review and will be explored in the following sessions: pediatric feeding disorders, family mealtimes, mealtime rituals, routines, and family dynamics, mealtime behaviors, barriers to mealtimes, parenting during mealtimes, parental stress during mealtime routines, interventions for pediatric feeding disorders, occupational therapy and pediatric feeding disorders, and parent training for mealtime strategies.

\section{Pediatric Feeding Disorders}

Pediatric feeding disorders are defined by the American Psychiatric Association's fifth edition of the Diagnostic and Statistical Manual of Mental Disorders (DSM-V-TR) as "a persistent (at least one month) failure to eat adequately and associated weight loss or significant failure to gain weight" (American Psychiatric Association, DSM 5 Task Force, 2013; Fishbein et al., 2016). The onset of the disorder occurs before six years of age. In many instances, children start receiving therapy for feeding difficulties using an interdisciplinary team in the neonatal intensive care unit. When an infant has an imbalance in the suck/swallow/breath synchrony, it can affect the child's progress toward developing self-feeding skills and overall development (Paul \& D’Amico, 2013).

Children with developmental delays such as Down Syndrome, Cerebral Palsy, Autism Spectrum Disorder, and other conditions are at an increased risk of developing PFD (Ledford \& Gast, 2006; Paul \& D’Amico, 2013; Yang, 2017). 40-80\% of children with developmental disabilities and $90 \%$ of children with ASD are diagnosed with a PFD. Children with ASD have difficulty with oral motor skills required to engage in the occupations of eating and feeding (Marshall et al., 2014). Frequently, they have difficulties with texture management and oral hypersensitivity, impacting feeding development (Marshall et al., 2014). 
The most frequently diagnosed feeding problem in children with ASD is food selectivity, described when a child has a limited food repertoire or high intake of a single food group (Berry et al., 2015). Limited food repertoire could include eating a specific type of food or rejecting one or more food groups. The food groups include fruits, vegetables, grains, protein, and dairy. Marshall et al. (2014) found that children with ASD tend to prefer foods with low nutritional value that contain simple carbohydrates, leading them to be overweight but malnourished. The lack of adequate dietary intake may also lead to diet-related medical complications such as obesity, poor bone growth, and constipation (Johnson et al., 2015). Restricted diets attribute to the physical, emotional, and cognitive deficits found in children with PFD and ASD. Marshall et al. (2014) found that children with ASD had a higher prevalence than typically developing children to develop at least one severe nutrient deficiency because of the restricted diets.

Pediatric feeding disorders present with similar underlying conditions which according to Goday et al. (2019), include_four domains. These four domains include medical, nutritional, feeding skills, and psychosocial. The medical features of the disorder present as significant weight loss, nutrition deficiency, and enteral feeding (Goday et al., 2019). Enteral feeding refers to feeding that occurs through a tube such as a gastrostomy tube or nasogastric tube (Fishbein et al., 2016). Nutritional factors include aspects of foods and nutrients consumed. According to Goday et al. (2019), when a child has an underlying condition relating to feeding skills, they have impairments in oral sensory functioning, oral motor functioning, and pharyngeal structures. Psychosocial factors include developmental factors, mental and behavioral health problems, social influences (i.e., caregiver strategies, mealtime expectations, cultural beliefs). Lastly, environmental factors include disruptive feeding environment, engagement of feeding and eating at school or places outside the home, responses to mealtime behaviors, inconsistent mealtime 
schedules, and food availability (Goday et al., 2019). Aside from the typical that underlie PFD, children with ASD may also display other physiological and behavioral components that must be considered to address the occupation of feeding.

Children diagnosed with ASD may exhibit sensory processing and sensory modulation difficulties, including hyperresponsivity and hypo-responsivity (Twachtman-Reilly et al., 2008). Children may display sensory seeking or sensory avoidant behaviors, which can impact eating processes. These behaviors affect how a child responds to the smell and taste of food. Some children may start showing preferences and avoiding certain textures and flavors (TwachtmanReilly et al., 2008). When a child displays sensory processing difficulties, another variable that should be considered is the physical environment in which the child engages in feeding. Noise, lighting, the smell of food, and the individuals present at mealtime_affect how a child engages in mealtimes. Some children may experience fight, flight, or fright responses depending on the environment (Twachtman-Reilly et al., 2008).

The involvement of the domains represents the complexity of treating a PFD. It takes a collective effort not only from all specialties to find treatments and interventions that best fit the child and the family, but also from the family to carry-over these techniques into the home environment. While it is important to carry over techniques into the home environment, the therapists in charge of the care of the child must take into consideration that families' mealtimes vary depending on their rituals, routines, and family dynamics.

\section{Family Mealtimes}

Mealtimes provide support, structure, and positively affect how family members interact and create memories (Ausderau et al., 2019). Feeding challenges can profoundly affect the child's development and cause tension between family members by enforcing changes in family 
routines and dynamics. These changes lead the family to have separate meals to accommodate the child with a feeding disorder (Ausderau et al., 2019). Although all parents experience stress during mealtimes, parents of children with feeding disorder and ASD have multiple variables that challenge the family's ability to successfully engage in mealtimes. Some of these variables include limited food repertoires, restricted diets, food refusal, and maladaptive behaviors (Ausderau et al., 2019).

Mealtimes shared with family members sitting around the table have been shown to provide excellent benefits for all individuals involved. Family cohesion is significantly improved when families maintain a regular mealtime routine (Jones 2018; Crowe, 2016). Other benefits include healthier eating habits, improved behaviors, better academic performance, and fewer antisocial behaviors (Jones, 2018). Family mealtimes provide children with a sense of belonging that can decrease the potential for the child developing high-risk behaviors.

A study by Fulkerson et al. (2006) as cited in (Jones, 2018), found that frequent family dinners may reduce the occurrence of high-risk behaviors in adolescents. High-risk behaviors include academic problems, eating disorders, depression, sexual activity, and violence (Jones, 2018; Utter et al., 2018; Harris et al., 2020). Regular family routines and rituals are also associated with increased social and emotional benefits, lower stress levels, increased parenting competence, and lower body mass index amongst parents (Utter et al., 2018). Crowe et al. (2016) found that family mealtimes for parents of typically developing children are a much less stressful routine than for parents of children with ASD.

\section{Mealtime Rituals, Routines, and Family Dynamics}

Families of children diagnosed with PFD and ASD experience changes in mealtime routines, family dynamics, and parental stress (Ausderau et al., 2019; Bahr \& Johanson, 2013; 
Crowe et al., 2016; Didehbani et al., 2011; Johnson et al., 2015; Postorino et al., 2015; Sharp et al., 2013; Suarez et al., 2014). Mealtime routines for these families are explicitly challenging due to the increased time spent planning, scheduling, and structuring mealtimes (Coldiron, 2016). Maladaptive mealtime behaviors disrupt the environment and family dynamics. The family's dynamics are ever-changing due to disturbances in daily routines, and it is within occupational therapy's scope of practice to focus on supporting these families. Learning how parents deal with these behaviors is essential for OTs because it can help create new strategies to be incorporated during evidence-based parent interventions.

Mealtime contains aspects of both rituals and routines, but these vary between families and cultures and contribute to their identity. The Occupational Therapy Practice Framework (OTPF) defines rituals as "sets of symbolic actions with spiritual, cultural, or social meaning contributing to the client's identity and reinforcing values and beliefs" (AOTA, 2014). The development of family rituals and routines are obtained throughout family life. The established rituals and routines provide the family with structure and support the child's early development, including language, academic, and social skill development (Spagnola \& Fiese, 2007). Just as it benefits the child, they also provide parents with parental efficacy and behavior monitoring. These rituals and routines lead families to create a sustainable overtime dynamic and must align with the family goals, values, culture, and child and parent characteristics (Spagnola \& Fiese, 2007).

Parent-child dynamics, along with rituals and routines, can positively or negatively influence mealtime routines. They both serve vital roles in maintaining the structure and family cohesion (Spagnola \& Fiese, 2007). Mother-child dynamics and father-child dynamics are essential and provide different benefits for the child. According to Aviram et al. (2020), the 
family dynamics differ for parents of typically developing children and parents of children with PFD. The mealtime dynamics of parents of children with PFD are related to the parent's perception of success, while parents of typically developing children are closely related to the child's characteristics. This highlights the importance of treating PFD with a holistic perspective and addressing mealtime behaviors and parental stress because these challenge parents in their effort to maintain a positive parent-child dynamic during mealtimes.

The benefits of having consistent mealtime rituals and routines are connected to parentchild dynamics. It is essential for the child to have consistent mealtime routines and rituals which must be carried out by parents (Aviram et al., 2020). Consistency across mealtimes, environments, parental feedback, and individuals present during mealtime, will allow for the child to create a positive routine around mealtimes. Parents of children with PFD spend a considerable amount of time trying to manage the feeding programs and maladaptive behaviors during mealtime. Having a positive mealtime routine will enable the parent to implement newly learned strategies in the home environment to manage mealtime behaviors.

\section{Mealtime Behaviors}

The mealtime structure provided by caregivers is essential in creating a healthy and positive feeding environment that enhances the child's healthy weight, eating, and behaviors (Berlin, Davies, Silverman, and Rudolph, 2009). Maladaptive and disruptive mealtime behaviors are highly prevalent in children with feeding disorders, which affects the family's engagement in mealtime routines (Aviram, Atazaba-Poria, Pike, Meiri, and Yerushalmi, 2015; Fishbein et al., 2016, Gonzalez \& Stern, 2016; Murphy, et al., 2020; Thullen \& Bonsall, 2017). Maladaptive/disruptive mealtime behaviors refer to tantrums, aggression, head-turning, throwing, and spitting food. As a response to the child's maladaptive mealtime behaviors, 
parents tend to create negative feeding strategies. Aviram et al. (2020) found that mothers of children with feeding disorders display adverse effects and intrusiveness during mealtimes. When a parent is continuously intrusive and struggles to control the mealtime routines, the child displays disruptive behaviors. Children may start showing food refusal, noncompliance, and temperament (Aviram et al., 2020).

As a result of the maladaptive and disruptive behaviors, parents treat food refusal and noncompliance by providing the child with their preferred food which reinforces the negative behaviors. Aviram et al. (2020) found that parent's behaviors during mealtime negatively impacts the child's behavior, limiting the ability for parents to engage in a positive mealtime routine free of negative practices. Another study reported that mothers' perceptions were perceived as anxious, controlling, and depressed during mealtimes, affecting the overall family function (Estrem et al., 2017). Parents need to maintain positive behaviors and be consistent during all mealtimes to provide the child with a supportive environment and an overall experience that is beneficial to the child.

To enhance the mealtime environment, a parent must set clear expectations for behavior and be consistent across meals and settings (Estrem et al., 2017). This requires the parent to learn about mediating roles, communication skills, and reinforcement approaches that can positively impact the mealtime routines. Often, parents receive training from the therapist in charge of their child's care that may assist with the learning process, but it takes trial-and-error to find the strategies that work best for families. A crucial step is knowing that the home environment can act as both a facilitator to mealtimes as well as a barrier. 


\section{Barriers to Mealtimes}

Although there are profound benefits to family mealtimes, there are also many challenges that may prevent the establishment and maintenance of routines (Suarez et al., 2014; Borowitz \& Borowitz, 2018). Jones (2018) found that the frequency of family dinners has decreased over the past decades. This may be because families are presented with barriers in everyday routines that may prevent them from engaging in family mealtimes successfully. Harris et al. (2020) found that parents' feeding practices can be both a barrier and facilitator to mealtime routines. Feeding practices are reinforced by the parent's goals, beliefs, and values. Simultaneously, some parents utilize a structured mealtime by being more responsive and attentive, leading to positive interactions between child and parent. Some may use a more controlling feeding practice that may lead to mealtime conflict (Harris et al., 2020). Some barriers to engaging in family mealtime routines include working schedules, extra-curricular activities, home environments, co-parenting arrangements, and socioeconomic status (Jones, 2018; Utter et al., 2018). There are also barriers to the parents feeding practices, feeding environment, and children's behavior during mealtime that can affect the child-parent relationship during mealtime routines.

The home environment can act as a barrier during mealtimes. The increased use of technology in the home can significantly impact the way families interact with one another. In the last decade, the use of smartphones and smart TVs has dramatically increased, leading families to spend less time communicating during mealtimes and increased time using the devices (Jones, 2018). Technology has significant impacts on the family's communication techniques and the family's health habits (Jones, 2018). Although there are many barriers to engaging in positive mealtime routines in the home environment, parents can also create, shape, 
and develop a healthy environment and routine by incorporating positive parenting styles and practices.

\section{Parenting During Mealtimes}

Parenting during mealtimes looks different for every household depending on the strategies that parents are incorporating, which may be based on the family's values and beliefs. Overall, parents have a common goal of getting the child to eat and explore different foods but often struggle to have constant control of the situation (Aviram et al., 2015). Parents often spend an increased amount of time during mealtimes preparing new foods, managing behaviors, or feeding the child, which can reflect mealtime behaviors in this population (Fishbein et al., 2016). Another challenge for parents is providing children with PFD mealtime opportunities outside of the home. Parents avoid eating outside the home and taking family trips because the change in schedule and structure of mealtimes is out of the child's routine. Parents have utilized some strategies to address their child's feeding problems, including setting up a strict mealtime routine, making the environment quiet and controlled, and changing foods and textures (Estrem et al., 2016). Having these strategies incorporated into the family's mealtime will help create and influence a positive routine that will benefit both the child and family.

Parents have a tremendous influence on their child, mealtime behaviors, and mealtime routines. Consistency across caregivers and feeding environment will assist in creating a positive mealtime experience for everyone involved. To do this, parents and other family members must communicate effectively and work as a team to deal with mealtime challenges together (Thullen \& Bonsall, 2017). Mealtimes are also influenced by parent feeding practices and styles, parental characteristics, and co-parenting situation. These components are essential to address because 
they create the overall family dynamic, and they can all affect children's behaviors and parental stress during mealtimes.

When parents model positive interactions and eating behaviors, it can encourage socialization for young children (Jones, 2018). Specific parent feeding practices and parenting styles can directly impact children's behaviors during mealtimes (Goodman et al., 2020). The traditional parenting style categories are permissive, authoritarian, authoritative, and uninvolved (Baumrind, 1991). Kiefner-Burmeister et al. (2016) and Roberts et al. (2018) found that parents who have an authoritative parenting style incorporate healthy feeding practices during mealtimes (Goodman et al., 2020; Jones, 2018). A study by Rigal et al. (2012) found that authoritarian and permissive feeding styles were strongly associated with children's feeding problems (Murphy et al., 2020; Estrem et al., 2017). This brings a key point that parent characteristics and anxiety regarding mealtimes may be inadvertently impacting the children's food aversions and refusal behaviors (Murphy et al., 2020; Estrem et al., 2017)

Often, parents fail to see how their characteristics such as parenting style, role, and skills can influence parent-child interaction and the child's behavior during mealtimes. Goodman et al. (2020) found that the interaction between parenting practices and children's eating during mealtimes is bi-directional, which means that children respond to parenting practices, and parents respond to their child's eating behaviors and habits. Feeding practices affect mealtimes and the parent's perception of their parenting style and overall competence (Aviram et al., 2015; Gonzalez \& Stern, 2016; Goodman et al., 2020; Murphy et al., 2020; Silverman Erato, and Goday, 2020). Chatoor et al. (2000), as cited in (Aviram et al., 2015), found that mothers' feelings regarding parenting inadequacy have been related to children's food intake. Specifically, 
Aviram et al. (2015) found that mothers who felt less competent as parents had more difficulty with control and were at an increased risk of conflict during mealtimes.

Parent competency may be related to co-parenting. Co-parenting is how parents relate to each other when facing specific challenges during their journeys and roles (Thullen and Bonsall, 2017). Positive co-parenting leads to improved communication and dynamics during mealtime routines. Thullen \& Bonsall (2017) found that parents who display better co-parenting techniques showed significantly lower stress when parenting children with ASD. During mealtimes, co-parenting is beneficial because both parents can help manage behaviors with consistency while also implementing behavioral strategies. Consistency of strategy implementation is particularly hard in single-parent households where one parent takes on all the responsibilities during mealtimes. In the United States, $23 \%$ of children under 18 live in singleparent households. This number is three times higher than the average of all countries (Kramer, 2020). Single-parent households are specifically vulnerable to stress due to the lack of support during mealtimes.

\section{Parental Stress During Mealtime Routines}

Mealtime challenges and disruptions, maladaptive behaviors, and feeding practices are all factors that are closely related to parental stress during mealtimes (Goodman et al., 2020; Murphy et al., 2020; Silverman et al., 2020). Gueron-Sela et al., (2011) found that parents of children with feeding problems have higher emotional stress, and this is exacerbated by children's health and developmental problems (Fishbein et al., 2016); Murphy et al., 2020; Silverman et al., 2020). Pedersen et al. (2004) as cited in (Fishbein et al., 2016), found that parents of children requiring enteral feeding reported higher stress than children with chronic 
disorders (Fishbein et al., 2016). Thus, feeding disorders often are associated with very high stress, regardless of whether that disorder is paired with comorbidities.

Children's maladaptive and disruptive behaviors have been shown to increase negative parental responses during mealtimes (Murphy et al., 2020). The study completed by Murphy et al. (2020) reported that the presence of maladaptive/disruptive behaviors during mealtime affects parental strategies/styles and feelings, which then affect the parent-child relationship and family dynamics. Silverman et al. (2020) found that a child's level of aggression and emotional regulation is closely related to caregiver stress. This study also showed that one of the main challenges parents face is not having the required skills to manage behavioral problems in the home environment. This brings on additional stress regarding the frustration associated with seeking parental training services due to the inability to cope and manage using only the available resources at home. Due to the prevalence of parental stress during mealtimes, it is vital to address all mealtime components to ensure an environment free of negative feeding practices. Parents need to receive services that provide a holistic approach that includes interventions for the child as well as the parents.

\section{Interventions for Pediatric Feeding Disorders}

Currently, children with pediatric feeding disorders may be referred to an interdisciplinary inpatient intensive feeding therapy program, outpatient feeding therapy clinic, home-based therapy, school-based therapy, or clinic-based therapy (Marshall et al., 2015; Gonzalez \& Stern, 2016; Ledford et al., 2018). Some clinics also provide services through telehealth. Intensive feeding therapy programs offer hospital-based interdisciplinary treatment that focuses on oral intake, oral motor skills, meal interventions, mealtime behaviors, and caregiver support/training (Gonzalez \& Stern, 2016). Children admitted to the program usually 
receive these services up to seven days a week, depending on the severity of their diagnosis. Typically, children admitted to an inpatient feeding therapy program are experiencing a higher level of severity, including gastronomy tube dependence (Fishbein et al., 2016). Treatment recommendations are aimed to be individualized based on the child's needs.

The intensive interdisciplinary feeding program provides excellent benefits for the child and the family. However, it places tremendous stress on the family because it requires the parents to commit to weeks of interdisciplinary treatment sessions, which involves a change in the family dynamic. Greer et al. (2008) found that initiation of the program may increase stress due to financial restraint and the expectations regarding the program and implementation of protocols at home. The study also found a significant reduction in stress from admission to discharge, which relates to the support received during the program. The support can be provided by all interdisciplinary team members and can assist parents in creating lifelong changes that will benefit the family overall. Occupational therapists provide services in all these settings through individual intervention and as part of an interdisciplinary team.

\section{Occupational Therapy and Pediatric Feeding Disorders}

Occupational therapy practitioners utilize the Occupational Therapy Framework:

Domain and Process to describe the concepts that describe OT practice (AOTA, 2017). Within the framework, the authors describe feeding and eating as areas of occupation. The occupations of feeding and eating are required for survival and human functioning across the lifespan (AOTA, 2017). Feeding is used to describe the act of bringing food to the mouth, and eating is defined as "keeping and manipulating food or liquid in the mouth and swallowing it" (AOTA, 2017). Feeding and eating are influenced by physiological, cultural, and environmental factors, including an individual's culture, rituals, routines, and engagement in social interactions. 
Feeding and eating are essential components of family mealtimes, and they play a role in the development of rituals, routines, and family identity (Fiese, Foley, \& Spagnola, (2006).

Occupational therapists can treat aspects of feeding disorder that pertain to mealtime routines, family dynamics, and parental stress. These include performance patterns such as family habits, routines, rituals, roles, cultural and personal contexts, and physical and social environments (AOTA, 2017). Because those feeding disorders have on the child's health, development, behavior, and interaction with family members, it is crucial for occupational therapists to address these components when developing a parent-based intervention. The prevalence of maladaptive behaviors, including tantrums, aggression, head-turning, throwing, and spitting food, can create additional challenges for parents and make it more difficult for them to implement mealtimes (Aviram et al., (2020).

In all settings, occupational therapy services address specific mealtime components and associated behaviors (Sharp et al., 2017). Behavioral interventions are addressed during these sessions by utilizing positive reinforcement for appropriate behaviors, shaping, and stimulus fading to facilitate positive responses (Howe \& Wang, 2013; Sharp et al., 2017). The occupational therapist often combines behavioral strategies with oral-motor therapy to decrease hypersensitivity to certain foods (Paul \& D'Amico, 2013). During these therapy sessions, parents are often asked to observe the interaction between the therapist and the child before implementing the strategies themselves (Sharp et al., 2017). When the parents are implementing these strategies in the clinic, the therapist is observing the parent-child mealtime dynamics and will subsequently provide feedback to ensure the parents understand the concepts (Aviram et al., 2020; Gonzalez \& Stern, 2016; Howe \& Wang, 2013; Sharp et al., 2017). Parent training in the 
clinical setting is crucial because parents must be able to apply learned mealtime strategies within the home environment to decrease stress.

\section{Parent Training for Mealtime Strategies}

Carry-over and generalization of mealtime strategies in the home environment and other settings are vital for treatment interventions to improve the overall feeding performance of a child with PFD. However, the services often only focus on a simple understanding of concepts rather than a true mastery and adoption of strategies (Marshall et al., 2015; Sharp et al., 2017). Due to this low level of specificity regarding parent training, parents have difficulty carrying over techniques learned in the clinic into other settings (Thullen \& Bonsall, 2017). Fishbein et al. (2016) concluded that although three-fourths or $75 \%$ of the referral PFD population were receiving therapy services, parents sought additional services to cope with additional stressors associated with feeding disorders. The family and environmental factors at home also limit the carry-over of strategies that can take place. These statistics are significant and show that parents need stress reduction techniques and mealtime behavior reduction strategies that can be incorporated during mealtimes in the home environment.

Occupational therapists have the knowledge and expertise to help parents manage maladaptive and disruptive behaviors to assist in lowering parental stress during mealtime routines. To successfully decrease parental stress during mealtime routines, occupational therapists can focus on parenting feeding practices, parenting styles, family dynamics, and home environment. Due to the lack of community resources, parents often must resort to trial-and-error to find a strategy that helps manage behaviors and stress during mealtimes (Gonzalez \& Stern, 2016). Fishbein et al. (2016) found that parents who had available resources did not necessarily see them as helpful. It was reported by the parents in this study, that the available resources were 
not necessarily beneficial when it came to managing behaviors and stress during mealtimes

(Fishbein et al., 2016). Occupational therapists can help parents successfully carry-over behavioral and stress reduction strategies by addressing family and environmental factors.

\section{Theoretical Support}

Occupational therapists (OTs) use the Occupational Therapy Framework: Domain and Process (OTPF) to help guide practice, interventions, and enable engagement in occupations. The OTFP alongside the occupational therapy theoretical models take into consideration the transactional relationship between the person and the environment (AOTA, 2014). Different components that are considered in the OTPF and within the theoretical models include the individual's culture, rituals, routines, social interactions, cultural, and social norms. The occupation of feeding and eating are essential for human functioning, it is influenced by physical, psychosocial, environmental, and social factors. Family mealtimes incorporate these components and when there is a disruption the mealtime dynamic is affected (Sharp et al., 2017. Occupational therapists have the expertise to address all these components to develop interventions that are family-centered by using the OTPF and theoretical models as guidance.

The models that align with the proposed qualitative research study are the Ecology of Human Performance model or EHP, and the Person-Environment-Occupation-Performance or the PEOP model. The EHP model focuses on the relationship between the individual's performance in a task/activity in relation to the context and environment (Cole \& Tufano, 2008; Hinojosa et al., 2017). Mealtime routines incorporate contexts that align with EHP such as cultural, physical, and social environments. It is essential to note that within the EHP model, there are aspects that must be addressed when evaluating or treating pediatric feeding disorders. In this case, the parents have specific role expectations that must be met within the social norms. 
Maladaptive mealtime behaviors can place a barrier on the achievement of role expectations and behavioral standards. By using the EHP model, the OT will ensure that all aspects of the person's environment and context are addressed when providing parent-based interventions.

The PEOP model is a client-centered model that focuses on the person-environmentoccupational transaction. The model includes four major constructs. The first construct includes the individual's intrinsic factors that can affect mealtime routines and parental stress such as, physiological, cognitive, psychological, and spiritual factors (Baum \& Christiansen, 2005; Cole \& Tufano, 2008). The second construct is made up of extrinsic factors such as, natural, and cultural environment, societal factors, social interactions, and social and economic systems. The third construct includes the persons' abilities, actions, tasks, occupations, and social and occupational roles. Lastly, the fourth construct is comprised of all constructs coming together to enable the individual to engage in meaningful occupations successfully. All these constructs are essential when addressing mealtime routines, mealtime behaviors, and parental stress. The parents intrinsic and extrinsic factors, and the environment can both positively and negatively impact family mealtime routines.

\section{Summary}

The literature review shows the importance of addressing mealtime behaviors and parental stress for parents of children with pediatric disorders, ASD, and related comorbidities. Pediatric feeding disorders are complex and affect every aspect of the child's life and the family and caregivers. Parents experience an overwhelming amount of stress during mealtime, closely related to changes in family dynamics, maladaptive behavior, and lack of community resources. Although children receive services for the feeding disorder and other comorbidities, these services do not focus on parental mastery places the parents under pressure to retain the 
information. The current literature focuses on the importance of interdisciplinary treatment to ensure that all aspects of feeding disorders are addressed to allow for better carry-over to the home environment.

The literature review identified a connection with parental stress and children with feeding disorders. Parental stress is higher for children with feeding disorders and other comorbidities such as ASD and developmental delays. Implementing a focus on parent training into the current interventions will improve the parent's ability to manage stress related to mealtime routines. Although the environment plays a significant role in treating pediatric feeding disorders, none of the interventions found throughout the literature review apply the EHP and PEOP models. The researcher intends to contribute to the existing literature regarding the use of specific occupational therapy theoretical models for treating parental stress related to pediatric feeding disorders. This is an essential area for occupational therapists to research to ensure that parents are provided with the appropriate support that enables the child to engage in the occupation of feeding successfully. The prevalence of mealtime behaviors and parental stress has inspired the author to gather more information regarding parent-used strategies to reduce mealtime behaviors and parental stress during mealtime. 


\section{Chapter III: Project Description}

\section{Introduction}

The purpose of this capstone is to identify and analyze how parents of children with PFD manage maladaptive mealtime behaviors and parental stress. This will be accomplished by interviewing parents of children with PFD at a hospital in a southern state. Parents of children with PFD experience changes in mealtime routines due to maladaptive mealtime behaviors and parental stress (Ausderau et al., 2019; Corwe et al., 2016; Johnson et al., 2015; Postorino et al., 2015). The prevalence of maladaptive behaviors such as tantrums, aggression, head-turning, throwing, and spitting increases the parent's vulnerability to stress during mealtime routines, which affect the family's positive engagement in mealtimes (Aviram et al., 2015; Fishbein et al., 2016; Gonzalez \& Stern, 2016; Murphy et al., 2020; Thullen \& Bonsall, 2017). Due to the bidirectional nature of maladaptive behaviors and parental stress, it is essential to learn the strategies used by parents to manage maladaptive behaviors and stress during mealtime.

The purpose of this qualitative research type project is to identify and analyze how parents of children with PFD manage maladaptive mealtime behaviors and parental stress. Parents will complete an interview questionnaire. The student investigator will derive themes regarding the most frequently used strategies for managing maladaptive mealtime behaviors and parental stress. The proposed project is essential to occupational therapy, the parents, and children with PFD because it will better support families in creating a positive engagement in mealtime participation. The significance of this research study is to fill the gap in knowledge regarding how parents manage maladaptive mealtime behaviors and mealtime stress to improve their occupational role of child rearing. 


\section{Objectives}

The proposed project will be supported by the following objectives:

\section{Learning Objectives:}

- Compare and contrast the current multidisciplinary interventions being implemented for children with feeding disorders

○ This objective will be measured by creating a reflection paper demonstrating the multidisciplinary interventions being implemented at a hospital in a southern state.

- Compare and contrast the literature to parental responses from interview questionnaire

- This objective will be measured by creating a reflection paper outlining the differences and similarities between literature and parental responses.

- Develop broader understanding of the application of interventions in the home environment

○ This objective will be measured by asking parents during weekly meetings how they implement different strategies learned in the clinic in the home environment. The findings will be reflected in a reflection paper.

- Evaluate the family characteristics (socioeconomic status, child's gender, number of children) that may impact frequency of the intervention implementation

○ This objective will be measured by gathering sociodemographic information during the interview questionnaire. The data gathered will be put into a chart and any other information will be available in the reflection paper.

- Describe advantages and disadvantages of current strategies used by parents to manage maladaptive behaviors and parental stress

○ This information will be gathered during parent interviews and the data will be available in chapter four. 
- Generalize themes from interviews and questionnaires that will help guide future interventions

○ Themes will be derived, and information will be reflected in chapter four.

\section{Project Objectives:}

- Introduce project to parents of children in the intensive feeding program

- This objective will be accomplished by handing out a participant information sheet at a hospital in a southern state.

- Interview parents of children with PFD

- This objective will be accomplished by completing in-person interviews.

- Transcribing interview data

- The student investigator will transcribe data verbatim

- Input data into Dedoose

- Analyze and report stressors parents experience during mealtime routines

○ This objective will be measured by gathering data from parent interviews and analyzing the most frequently occurring stressors during mealtime routines.

- Analyze and report reduction techniques to improve parent-child interactions

- This objective will be measured by gathering data from the parent interviews and analyzing the most frequently used stress reduction strategies and how they impact parent-child relationships.

- Analyze and report most frequently occurring maladaptive behaviors and the strategies used by parents to manage these behaviors

$\circ$ This objective will be measured by gathering data from the parent interviews and analyzing the most frequently occurring maladaptive mealtime behaviors.

- Analyze and report the similarities and differences of strategies used by families 
○ This objective will be measured by gathering data from the parent interviews and analyzing the similarities and differences between parental responses.

- Attending weekly meetings with site mentor

- Share study Findings with facility

- Create a poster for dissemination using USAHS template

- Write Chapters 4 \& 5 of the Capstone paper

- Submit completed and approved project

\section{Process and Methods}

\section{Research Design}

A qualitative research design was chosen to gather information from parents' firsthand experiences during mealtime routines. This research design will utilize an open-ended interview style questions to be administered face-to-face. The data collected from the open-ended interview questions will provide the student investigator with potential themes to provide the reader with an insight into how families manage behaviors and stress during mealtime.

Interviews are a vital step in collecting data on human perception to guide the development of future applicable interventions or studies related to parental stress management with children who have PFD.

The qualitative research project consists of an interview questionnaire developed and administered by the student investigator. The interview questionnaire has three sections: mealtime routine, mealtime behaviors, and parental stress. The qualitative research project will hope to answer the following research questions: (1) how do parents of children with PFD perceive maladaptive behaviors during mealtime? (2) how do parents of children with PFD 
describe strategies to manage maladaptive behaviors during mealtime? And (3) how do parents of children with PFD view their roles when managing stressors during mealtime?

\section{Participants}

The project will consist of anywhere from five to twenty parents of children diagnosed with Pediatric Feeding Disorders between the ages of twenty and sixty years old. The participants will be recruited from a hospital in a southern state. A snowball and convenience sampling method will be used. Participants will be handed out a participant information sheet along with a flyer, and individuals who choose to participate and meet the inclusion criteria will be included in the study. The inclusion criteria include parents or primary caregivers of children diagnosed with PFD between the ages of twenty and sixty years old. Parents of children who do not have a PFD diagnosis will be excluded from participating in this study. Participation in the research study is voluntary, and participants may withdraw at any time. The participants will be required to sign a physical copy of the consent form in blue or black ink. The informed consent form includes the risks, benefits, and compensation (none) to be reviewed by the participant.

\section{Informed consent}

Please find informed consent in Appendix B

\section{Recruitment Procedures}

The interviews and recruitment will take place in-person at a hospital in a southern state. The hospital offers an intensive feeding program that focuses on behavior management, food presentation, and tolerance to foods. The program is offered three times per day, five days per week, for a total of eight weeks. The parents participating in the research study will be required to fill out a questionnaire and assessment, taking sixty minutes; only one session is required. Participants will be handed a participant information sheet and research study flyer. Individuals 
who meet the inclusion criteria and choose to participate will physically sign an informed consent in blue/black ink. Once the informed consent is signed, individuals will set up a time with the student investigator to complete the questionnaire and assessment. The student investigator will then obtain and input data into the data analysis tool Dedoose where a data analysis of information provided by the participants will be completed.

\section{Instrumentation}

The instruments used to collect data for this qualitative research study include an interview questionnaire. The student investigator created the questionnaire with feedback from the student's mentor as well as information from previous research studies. The interview questionnaire will include open-ended questions, yes/no questions, semi-open questions. Participants will have the opportunity to explain further for specific questions.

\section{Data Collection}

The participants will be interviewed individually at a hospital in a southern state. The interview audio will be recorded to ensure that the transcription of data is accurate. The interview questionnaire consists of a mealtime routine, mealtime behavior, and parental stress sections. Please see the interview questionnaire in Appendix C.

\section{Data Analysis}

Following the data collection, the student investigator will complete the transcription of the data provided by the participants during the interview. The computer software, Dedoose will be used to assist with the data analysis. Following the data transcription, the student investigator will type up any additional notes and sort and arrange all information into categories that make it easily accessible (Creswell \& Creswell, 2018). The student investigator will then derive themes from the parent responses to identify the strategies utilized most frequently to manage mealtime 
behaviors and parental stress. All data will be presented in the final product of the qualitative study alongside specific quotations. The data will be destroyed after three years following the completion of the study.

\section{Conceptual Framework: Theory of Change Diagram}

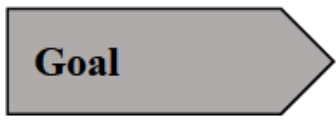

\section{Project Objectives}

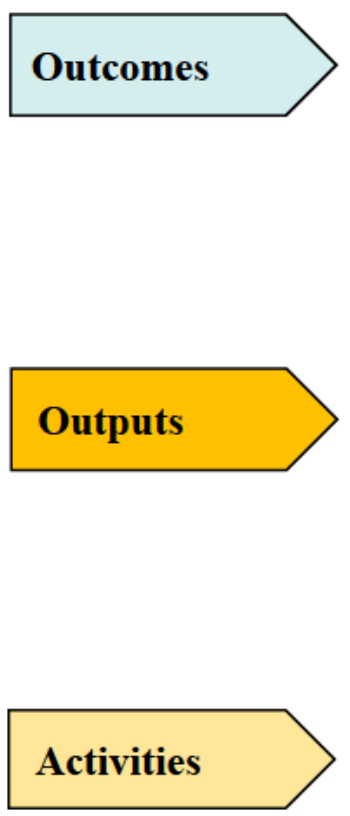

\section{Timeline}

Identify OT strategies to assist parents with managing maladaptive behaviors and decreasing overall parental stress during mealtimes with children diagnosed with PFD
1. Analyze and report stressors parents experience during mealtime routines

2. Analyze and report reduction techniques to improve parent-child interactions

3. Analyze and report most frequently occurring maladaptive behaviors and the strategies used by parents to manage these behaviors

4. Analyze and report the similarities and differences of strategies used by families
Develop parental strategies to appropriately handle maladaptive behaviors for children with PFD

\section{$\uparrow$}

Parents who have children with PFD attending the feeding program or outpatient feeding therapy at a hospital in a southern state who are willing to participate in the

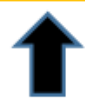

Interview parents of children with PFD at a hospital in a southern state. These interviews will identify the following: (1) how do parents of children with PFD perceive maladaptive behaviors during mealtime?

(2) how do parents of children with PFD describe strategies to manage
Learn how OTs address parental stress related to maladaptive behaviors during mealtime among parents with children with PFD

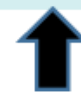

Children with PFD who are attending the feeding program or outpatient feeding therapy at a hospital in a southern state. The OT's who provide treatment.

Experiential time at a hospital in a southern state will include observing children with PFD while receiving occupational therapy and feeding therapy services and identifying specific maladaptive behaviors that occur during mealtimes. 
The proposed research project will take place at a hospital in a southern state. The capstone experience will be completed from January 2021 until April 2021. The experience will incorporate time with parents completing interviews and assessment as well as observations of therapy sessions, team, and parent meetings.

\section{Timeline}

Weeks

Weeks 1-5

\section{Weeks 6-12}

Weeks 13-15

\section{Week 16}

\section{Tasks}

- Meeting with feeding team

- Observation of feeding therapy sessions

- Observations of interdisciplinary feeding therapy sessions (SLP and PT)

- Early recruitment of participants

- Weekly parent meetings

- Learn about protocols, techniques, approaches to feeding through documents, webinars, research articles, and resources provided by site supervisor

- Interview parents/data collection

- Observation of feeding therapy sessions

- Observation of parent-training

- Meetings with feeding team

- Learn about Dedoose

- Transcription of data

- Learn about protocols, techniques, approaches to feeding through documents, webinars, research articles, and resources provided by site supervisor

- Analyze qualitative data from interviews

- Derive themes from the information provided by parents

- Debriefing session with parents and feeding team

- Write chapter 4 and 5

- Complete write ups

- Create poster 
- Submit completed project

\section{Conclusion and Next Steps}

Current literature shows the need for holistic interventions that focus on stress management during mealtimes and parent-based interventions to manage maladaptive mealtime behaviors during mealtime routines (Thullen \& Bonsall, 2017). Curtin et al. (2015) found that little research has been done to connect the relationship between feeding disorders and mealtime behaviors. Although children are receiving services for the feeding disorder, the interventions are not focused on parental proficiency in skills to incorporate in the home environment, making it difficult to carry over techniques.

The proposed research study will fill the gap in knowledge regarding strategies used by parents to manage mealtime behaviors and parental stress. The project will utilize the EHP and PEOP models to help guide the parent-directed interviews because they both encompass social, cultural, and environmental aspects of mealtime routines. The project will focus on strategies that parents are currently incorporating into mealtime routines because it will assist with the clinic's carry-over to the home environment. Following the completion of the project, the student investigator will disseminate findings to Feeding Matters, a national parent support group. 


\section{Chapter IV: Results and Analysis}

\section{Introduction}

The primary goal of this qualitative research study was to assess how parents of children with pediatric feeding disorders manage behaviors and stress during mealtime routines. The study was conducted at a hospital that offers an intensive feeding program and outpatient feeding therapy for children with pediatric feeding disorders. The intensive feeding program lasts eight weeks and provides interdisciplinary services (OT, SLP, feeding therapy, and PT). This study collected information through in-person interviews of parents of children with a pediatric feeding disorder diagnosis. The interviews were recorded to ensure accuracy during the transcription of data.

The purpose of this capstone project was to assess how parents of children with Pediatric Feeding Disorders manage behaviors and stress during mealtime routines. Parents of children with PFD were interviewed using a questionnaire created by the student investigator and project mentor. The goal of the questionnaire was to gather information regarding mealtime routines, mealtime behaviors, and parental stress during mealtime to enhance the role of occupational therapy in supporting parents. The problem is that few research studies show how parents manage behaviors and stress during mealtime routines.

The qualitative research project was hoping to answer the following research questions regarding maladaptive behaviors and parental stress during mealtime routines: (1) how do parents of children with PFD perceive maladaptive behaviors during mealtime routines? (2) how do parents of children with PFD describe strategies to manage maladaptive mealtime behaviors during mealtime? (3) how do parents of children with PFD view their roles when managing stressors during mealtime? Within this chapter, we will discuss the project objectives, and 
research questions, process of collecting data, participants and recruitment procedures, statistical data, developed themes, and evidence of trustworthiness. The results and analysis detail the most frequently occurring mealtime behaviors and the strategies parents utilize to reduce stress during mealtime routines. The following section will explain the project data collection guidelines.

\section{Data Collection}

The project timeframe consisted of 10-weeks from January 2021 to March 2021 of onsite observation at a Hospital in a Southern State. The data collection occurred in weeks four through six. During the ten weeks, the student investigator observed children undergoing intensive feeding therapy for a feeding disorder and outpatient feeding therapy. The intensive feeding therapy program provided interdisciplinary services, and the team consisted of occupational therapists, speech therapists, and physical therapists. Each child received these interdisciplinary services from eight in the morning until twelve-thirty in the afternoon from Tuesday to Friday. The team attended weekly meetings to discuss each patient's progress to ensure everyone worked toward the same goal.

Data was collected during in-person interviews. The interviews lasted from forty-five minutes to an hour, depending on parental responses. General demographics information was gathered such as, child's age, sex, number of individuals living in the household, mother's age and employment, father's age and employment, current diagnoses, number of children and birth order, and what services the child is currently receiving. The interview questionnaire consisted of a mealtime routine section, mealtime behavior section, and parental stress section.

The participants were recruited using convenience sampling. Parents or primary caregivers of children with a PFD diagnosis receiving services at a hospital in a southern state were approached and handed out a participant information sheet. The individuals who were 
interested in participating in the study made an appointment for an interview with the student investigator. The interviews took place when the child was receiving services, whether in the intensive feeding program or general outpatient therapy. Five participants volunteered to participate in the study, two males and three females. Four of the participants were parents, and one participant was a grandparent who was the primary caregiver. The sample of individuals who participated was like the individuals described in chapter 2. All children of parents who participated had a PFD diagnosis.

The main contributor to whether a parent would volunteer in the study was time. Parents in the intensive feeding program were at the clinic from eight in the morning until twelve-thirty in the afternoon from Tuesday to Friday. The only time parents were available was when their child was in a session, making it more complicated to schedule the interview. The interview took fortyfive minutes to an hour, and each therapy session lasts thirty minutes. To ensure that the interview could be completed in one sitting, the interview was scheduled when the child had back-to-back sessions. The outpatient clinic is located within a hospital and the parents/children must go in through the hospital's main lobby to reach the outpatient clinic. Due to COVID-19, one of the interviews had to be completed outside the clinic because the participant did not feel comfortable going into the hospital. The following section will explain the outcomes of the parent interviews

\section{Objectives and Deliverables:}

- Compare and contrast the current multidisciplinary interventions being implemented for children with feeding disorders

$\circ$ The intensive feeding program at a hospital in a southern state offers a multidisciplinary approach. The children currently enrolled in the program have other comorbidities and by having a multidisciplinary approach, the therapists can 
address all components that are affecting the occupations of feeding and eating. All children are evaluated by each profession (OT, SLP, PT), and they all work collectively to find treatments and interventions that benefit the child and the family.

○ During my observations with OT the interventions that were being implemented included: improving oral motor skills, exploring new foods/textures, SOS approach, sensory/tactile desensitization, and sensory play with foods. The speech therapist was focusing on tongue lateralization, swallowing, reflux, and dysphagia. The physical therapist focuses more on treating posture, core strength, and treating the patient's physical comorbidities that may be affecting feeding. All therapists attend a weekly meeting where they discuss the patient's goals, progress, and plans for future sessions.

- Have a broader understanding of the application of interventions in the home environment

- Parent A: Dad oversees taking child to sessions and can implement some of the strategies in the home environment. However, the child has just started attending daycare on the days that he is not attending the program. Father mentions that at the daycare, the person in charge of feeding him must take care of two other children and it may take her up to 45 minutes to feed him. He also mentions that mom and grandmother are a little bit more lenient with their approach at home.

o Parent B: Dad oversees taking child to feeding therapy sessions. Dad mentions that his child displays maladaptive behaviors during mealtimes including gagging, crying, and pushing food away. These are all behaviors that he does not display during feeding therapy. Dad mentions that he is usually continues feeding the child 
as long as he is not aspirating, but wife stops feeding when child displays maladaptive behaviors, and in turn reinforcing the negative behaviors. Dad will demonstrate strategies learned to the mother to ensure that they are both on the same page.

○ Parent C: Mom oversees taking child to sessions. Mom mentions that her child is doing well during feeding therapy but will not incorporate new meals at home, which is the hardest part about carrying-out techniques into the home environment. She has purchased the new foods that the child enjoys but mentions that child continues eating the same preferred foods. Mom is buying a calendar/journal where the child can keep track to new foods and can try adding at least one new food every week.

- Compare and contrast the literature to parental responses from the interview questionnaire

- Research studies show that disruptions in mealtime routines such as mealtime behaviors are related to increased stress during mealtime (Doodman et al., 2020; Murphy et al., 2020, Silverman et al., 2020). The data from the current study shows that parents of children with PFD experience increased stress during mealtimes. The average daily stress for parents is 6.8 on a scale from 1 to 10 . The average stress during mealtime routines is 7.4 on a scale from 1 to 10 . These numbers align with the literature review that outlines that parent experience increased stress during mealtime routines. The current study also shows that when parents have a co-parent or another caregiver it helps create consistency around mealtime and it helps reduce the stress that surrounds mealtime routines. 
- Determine the family characteristics (socioeconomic status, child's gender, number of children) that may impact frequency of intervention implementation

○ Parent A: This child is currently completing the second round of the intensive feeding program and the father has been noticing improvements at home. Parents are currently working less hours a week to be able to take the child to intensive feeding sessions. Both parents in the household are employed and have insurance to cover the intensive feeding program.

O Parent B: Parent feels grateful to have the opportunity to have child in the intensive feeding program, but it causes a financial strain on the family because they have a high out-of-pocket deductible. The parent must work the second shift (from two in the afternoon until midnight) to be able to take the child to intensive feeding sessions.

O Parent C: Patient is from the West Coast of Florida and travels every Monday to Boynton Beach so that the child can participate in the intensive feeding program. Prior to starting the intensive feeding program, the parent had to quit her full-time job and find an apartment in Boynton Beach, FL. The mother reports that this has been difficult, and it has caused significant stress in her family, although she is grateful her child is making improvements. Without Medicaid, the family would not be able to afford this feeding program.

- Describe advantages and disadvantages of current strategies used by parents to manage maladaptive behaviors and parental stress

$\circ$ During the interviews, every parent reported that when their child is displaying maladaptive mealtime behaviors such as tantrums, aggression, head-turning, 
throwing, and spitting food, they ignore the behavior. However, $80 \%$ of parents report that this does not help and sometimes it leads to their child's behavior getting worse. When the behavior gets worse, parents inadvertently reinforce the negative behavior by taking the child out of the highchair or taking the non-preferred food away.

- Regarding how parents manage stress, four out of five $(80 \%)$ of parents reported that talking to family and friends was a way of decreasing stress as well as using outdoor activities such as walks, runs, and visits to parks to relieve stress. The one limitation that parents report is not having enough time to get every day outdoors. Three out of five parents (60\%) also report using other methods such as listening to music, meditating, and cleaning as methods to reduced stress brought on by mealtime routines.

- Derive themes from interviews and questionnaires that will help guide interventions

○ Main themes: Mealtime behaviors are highly stressful, ignoring behaviors during mealtimes, and managing stress independently from mealtime.

\section{Project Analysis}

Three research questions support this qualitative research study: (1) how do parents of children with PFD perceive maladaptive behaviors during mealtime routines? (2) how do parents of children with PFD describe strategies to manage maladaptive behaviors during mealtime? And (3) how do parents of children with PFD view their roles when managing stressors during mealtime? The student investigator hypothesized that parents perceive mealtime as more challenging due to the presence of maladaptive mealtime behaviors. It is also hypothesized that parents will describe managing behaviors in the form 
of negative or positive reinforcement, punishment, or rewards. Lastly, it is hypothesized that parents view their daily roles as overwhelming when dealing with stress during mealtimes. After the data collection and after further analysis, these research questions and hypotheses were proven to be true. From the research questions and the interview questionnaire, the student investigator was able to derive themes to show data representation. The following section will outline the data analysis, derived themes, and specific excerpts from the interview questionnaire.

\section{Data Analysis}

Data analysis consisted of collecting data through in-person interviews. The student transcribed the data from the interviews verbatim to ensure accuracy of information. The data was analyzed using Dedoose. Sociodemographic information of the parents who participated in the study was collected to identify how these can affect mealtime routines and parental stress during mealtimes. The information is presented below in Table 1 .

\section{Table 1}

Sociodemographic of Parents of Children with PFD

\begin{tabular}{r|lllll}
\multicolumn{1}{c}{ ID } & ID 1 & ID 2 & ID 3 & ID 4 & ID 5 \\
\hline Child's Age & 6 & 16 & 2 & 8 & 3 \\
Child's & Male & Female & Male & Female & Male \\
Gender & & & & & \\
Ethnicity & White & Hispanic & Hispanic & Hispanic & Hispanic \\
Primary & English & Spanish & English & Spanish & English \\
Language & & & & &
\end{tabular}




\begin{tabular}{r|lllll} 
Mother's & Full-time & Unemployed & Full-time & Full-time & Full-time \\
Employment & Student & & & & \\
Mother's Age & 36 & 46 & 36 & 41 & 39 \\
Father's & Full-time & Full-time & Full-time & Full-time & Full-time \\
Employment & & & & & \\
Father's Age & 33 & 47 & 39 & 38 & 37 \\
Family Size & 4 & 3 & 3 & 6 & 5
\end{tabular}

\section{Mealtime Routine}

For the first section of the questionnaire mealtime routines were explored. The goal of this section is to get to know the family routine and how the family environment can impact mealtimes. Consistency during mealtime will assist in creating a positive mealtime routine for the child and the family. The mealtime routine section of the interview questionnaire included the following questions:

\section{Table 2}

\section{Mealtime Behavior Section}

1. How many times a day does your child eat?

2. Do you think your child eats enough throughout the day?

3. Does your child eat at the same time every day?

4. Does your child let you know when he/she is hungry?

5. Do you have concerns regarding your child's weight?

6. does your child self-feed?

7. How long does it usually take your child to eat a meal/to feed your child?

8. Are any meals on the day family meals?

9. What meal does your child do best with in relation to time, and behaviors?

10. During mealtimes, are there other activities going on in the area when your child is eating? 11. And does your child eat the same or different across different settings? 
Overall, there were some similarities in the answers given by the participants. When asked if the child eats at the same time every day, three out of five $(60 \%)$ of parents answered "yes". One parent reported that setting a routine was critical, stating: "yes, we have to be very punctual because of his medications." Another parent reported, “Almost always, depends on if he sleeps a little bit more, but realistically he has a pretty stable routine." Another parent stated, "yes, he already has a routine.." One of the parents that reported whose child did not have a mealtime routine stated, "no, sometimes I have to follow her around so she will eat." One of the parents reported that setting a specific mealtime routine is difficult "because sometimes we are here (in therapy) until 5 o'clock and we don't get home until 5:30 on Tuesdays Wednesdays and Thursdays. " It is important for these families to set up routines, especially when parents must cook different meals for their children.

Aside from setting routines, another aspect of mealtimes essential for families is whether they eat the same food as other family members. When asked if the child eats the same food as the rest of the family, five out of five (100\%) reported that parents must make different meals for the children with PFD. One parent reported, "Absolutely not, and if we go out of restaurants, he won't eat at all." Another parent reported, "no, it is very hard because of his G-tube; we have to count the calories of his purees." Another parent stated, "I have to prepare something else to make sure she eats" One parent reported, "He has separate meals because my daughter is a picky eater, and so my wife prepares different foods for the kids. I think my wife is at the point where she just wants them to eat" Lastly, one parent reported that their child eats the same protein. Still, she must make separate sides "the sides that's one of the issues that we have with him; he doesn't want to eat any vegetables; if I make him baked beans every single meal, he will 
eat baked beans every meal." Parents are taking extra time to prepare meals that their children will eat and spend additional time ensuring they finish their meals.

Another essential aspect of the mealtime routine is the amount of time children spend eating and the amount of time parents spend trying to feed their children. Out of the five children whose parents participated in the survey, two of them self-feed, and three require total assistance for feeding. When asked how long does it usually take your child to eat or to feed your child? Two out of five (40\%) parents reported that it takes their child between five to thirty minutes to eat a meal. Three out of five $(60 \%)$ parents said that it takes thirty to forty-five minutes for a child to eat or to feed the child a preferred meal. One parent stated, "If I can get him to eat, it takes him about a good maybe like a half hour to 45 minutes to finally get him to eat the food. Dinner, that's a fun one, it usually takes me about an hour to get him to eat a full you know his plate of dinner." Another parent reported, "for the most part, breakfast is pretty fast because it always the same thing, an ice cream shake, so it takes around 10 minutes. Dinner, I would say around 30-minutes". Increased time spent preparing meals and time spent feeding the child or getting their child to eat has the potential to increase parental stress. Table 2 displays the time it takes a child to eat a meal.

\section{Table 3}

Time Spent Eating

Participant ID 1

Participant ID 2

Participant ID 3
5-30 minutes

5-30 minutes

30-45 minutes 


\section{Participant ID 4}

Participant ID 5
30-45 minutes

30-45 minutes

\section{Mealtime Behavior}

For the second section of the questionnaire, mealtime behavior was explored. This section aims to gather information regarding a child's behavior and the most frequently occurring mealtime behaviors. The interview questionnaire's mealtime behavior section consisted of the following questions:

\section{Table 4}

\section{Mealtime Behavior Section}

1. Does your child have any behavior issues?

2. Does your child have any attention difficulties?

3. Does your child usually enjoy eating?

4. Does your child have difficulty with sensory processing? (i.e., noises, textures, clothing, touch, movement, lights)

5. Does your child display any of the following maladaptive behaviors during mealtime?

6. What does your child do when a non-preferred food is offered?

7. Do you use any strategies to help your child eat? (i.e., positive reinforcement, behavioral reinforcement, behavioral techniques, reward system)

8. Please describe other concerns about your child's eating behavior or emotional condition?

Five out of five $(100 \%)$ parents reported that when presented with non-preferred foods, the child will push the food away. In addition to that, a parent stated, "something that he does always when non-preferred food is offered is made like a puking/gagging noise/nasty expression. Without knowing, smelling, trying, nothing. " Another parent reported that their child would push the food away and "If she gets a handle on the food, she will throw it." When asked if their child has any behavior issues, three out of five (60\%) answered yes. One parent stated that "during dinner, if it is something that he doesn't want to eat or I put something new on his plate, he will start going into meltdown where he is starting to cry." When asked if their child enjoyed eating, 
five out of five parents reported that their children enjoy eating if they are eating preferred foods.

\section{Ignoring Maladaptive Mealtime Behaviors}

The interview's mealtime behavior section provided insight to answer the following research question: how parents of children with PFD describe strategies to manage maladaptive behaviors during mealtime routines. A theme that developed from parental responses is that parents of children with PFD report ignoring maladaptive mealtime behaviors. Waiting for the behavior to stop was the most frequently used strategy used by parents.

When the child displays maladaptive mealtime behaviors, five out of five (100\%) parents reported that they let the behavior pass and ignore the behaviors. One mother reported, "I have opted for letting him be when he feels like that until he feels better, and it works." One father reported, "I let him get through it; I am more with the mindset that he has to get through those things, so I let him." This parent also stated that when ignoring the behaviors does not work, "I sometimes ignore him, sometimes I soothe him, and wait for him to relax." The other parent reported, "I just kind of ignore any behaviors. We are at the point where we just ignore it." Aside from ignoring behaviors, one of the parents used punishment when their child displayed maladaptive behaviors; this parent stated, "I tell her that if she doesn't finish eating or if she continues to behave bad, she won't get her phone or tv.." Another parent tries to use positive or negative reinforcing techniques when their child displays maladaptive behaviors during mealtimes, "I try to reinforce him, "Buddy, I need you to eat so that you can get big and strong so you can play sports, or if you don't have to play sports, you can do karate, or whatever, or so you can help mommy when mommy gets old.” Figure 1 represents the most frequently occurring mealtime behaviors. 


\section{Figure 1}

\section{Mealtime Behaviors}

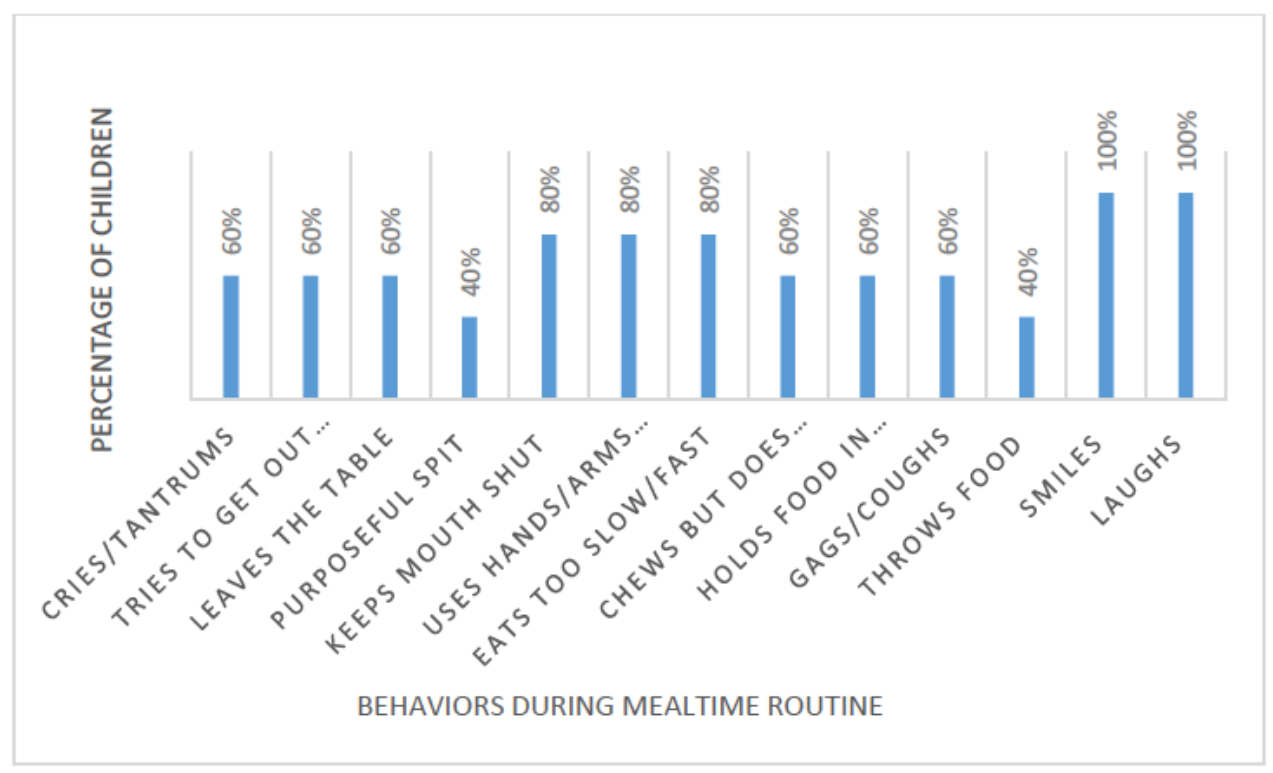

\section{Parental Stress}

For the last section of the questionnaire, parental stress during mealtime was explored.

This section aims to gather information regarding parental stress and how the parents report their feelings regarding the support from co-parents, family, and therapists. The interview questionnaire's parental stress section consisted of the following questions:

\section{Table 5}

Parental Stress Section

1. Do you enjoy mealtimes with your child?

2. Do you have a co-parent, or is there another caregiver involved?

3. When you have difficulties with the care of the child, I seek help or advice.

4. What do you do when your child displays maladaptive behaviors?

5. Have you noticed increased stress during mealtimes?

6. Which of the following methods do you use to reduce stress?

7. Have you found any of these strategies helpful?

8. Are you able to implement feeding strategies learned during feeding therapy in the home environment? 
9. Do you face any challenges when implementing these strategies? If so, what are they? 10. What suggestions have worked?

11. What suggestions have not?

12. Do you feel overwhelmed in the parenting role?

13. Do you find it difficult to balance the parenting role with other responsibilities?

14. What is your daily stress level from 1-10?

15. What is your stress level during mealtime from 1-10?

16. Have you told your OT about challenges during mealtime?

When asked if parents enjoy mealtimes with their child, three parents $(60 \%)$ reported that mealtimes are a major source of stress, and they did not enjoy mealtimes. One parent stated, "We have gone through some ups and downs. We have had some harder. With him, it is kind of like we need to breakthrough. In order to stop using a phone or something like that. And when he is throwing tantrums, it's rough." Another parent reported, "In reality, no, it is very stressful. He mortifies me a lot; he does not do it with anyone else but with me." One parent reported that mealtimes are only enjoyable when the child is eating preferred foods, "Yes, as long as he is eating preferred foods, " but when he is presented with non-preferred foods, it causes immense stress. Two of the parents that enjoy mealtimes with their child reported, "I do because I am trying to do my best to get him to eat and everything like that and I want him to have a positive mealtime." The other parent stated, "I enjoy watching her eating because I see an improvement every single day. But it is stressful because I sometimes do not get to eat because I am worried about her eating." Although some parents enjoy mealtimes with their children, they are still under considerable stress because their child is not eating preferred foods and because mealtimes can be unpredictable.

During the interview, parents were asked if they had a co-parent, or another caregiver involved in the care of the child. Five out of five parents reported having a co-parent or caregiver that assists with the child's care, making it easier for the family to manage throughout the day. 
Five out of five parents reported seeking advice from therapists, family, friends, and spouses when having difficulties with the child's care. Five out of five parents also say being able to implement feeding strategies learned in the clinic in the home environment. One parent stated, "I try to implement some of the strategies, but I have gotten to a point where I just want her to eat, so I just do what I can" Three out of five parents, however, report that time is the main challenge when implementing the strategies. One parent stated, "Yes, time. I work the second shift, so my wife is in charge of dinner, and I am not able to be there. But we try our hardest." Another parent stated, "Yes, I have tried everything the therapists have told me to do. It is especially difficult because he is a teenager" Parents who participated in this study are constantly taking advice from therapists and implementing strategies at home as much as possible.

\section{Mealtime Behaviors Viewed as Highly Stressful}

The interview's parental stress section provided insight to answer the following research question: How do parents of children with PFD perceive maladaptive mealtime behaviors? A theme that developed from this question was that parents perceive maladaptive mealtime behaviors as highly stressful based on the results from questions one, five, and fourteen in the parental stress section.

When asked if the parent has noticed increased stress during mealtimes, five out of five parents reported feeling increased stress during mealtimes. One parent reported, "When I know the feed is coming, it's stressful because I don't know how he is going to do, I don't know if he is going to have a tantrum, or if it's going to go smooth. Leading up to it, it's stressful; once we start and he's doing well, then it's fine". Another parent stated, "yes, of course". One parent reported, "Yes, because its constantly trying to "I need you to come sit back down, I need you to eat your food" and now has to also worry about a sibling who 
is also displaying the same behaviors "then I turn around, and I have my daughter start, like "no, I don't want it". So that increases the stress, because I do not want to deal with low weight with two kids". Another parent reported that although she is happy when the child is eating, "Yes, my stress increases when she doesn't eat". Lastly, one parent noted that she is getting used to stress because she has been dealing with PFD and behaviors for a long time "yes, I get stressed when he doesn't behave and wants to complicate things. I am very used to it; it has been many years". Figure 2 represents the parents' daily stress and stress during mealtimes on a scale from one to ten.

\section{Figure 2}

\section{Parental Stress}

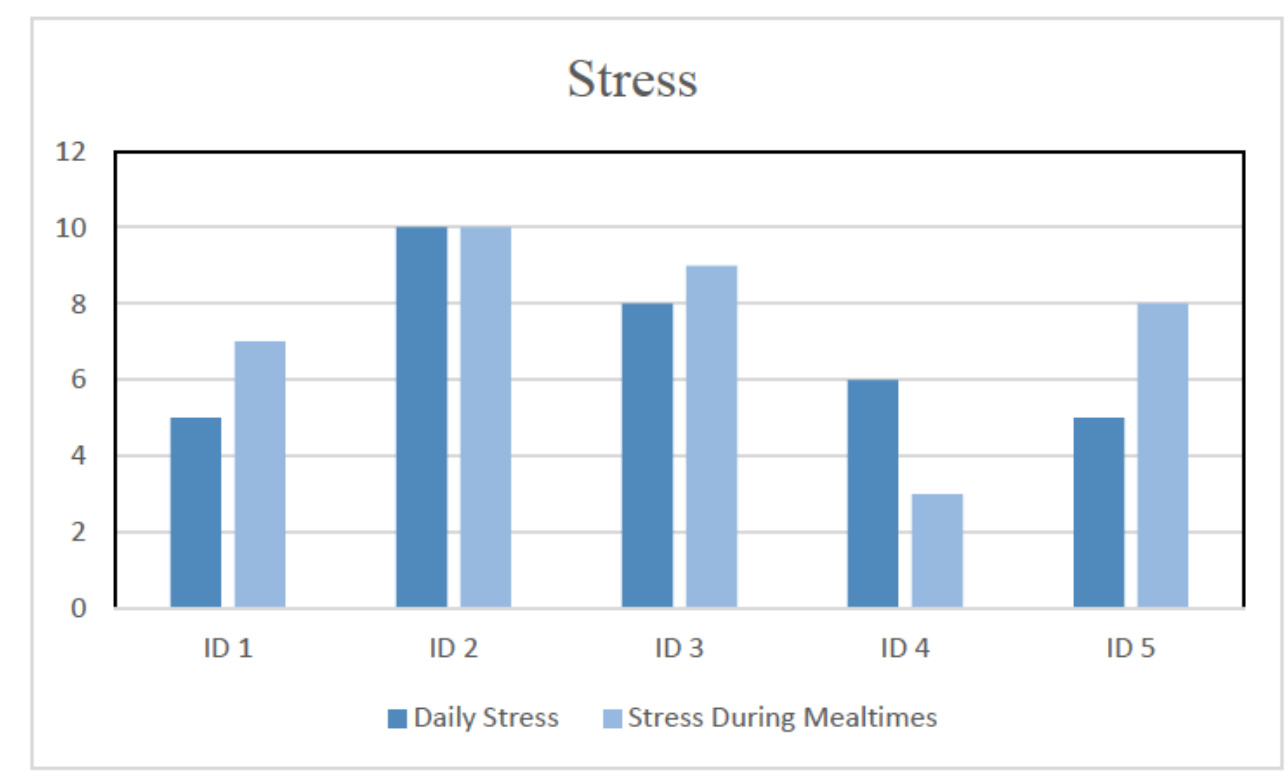

\section{Managing Stressors Independently from Mealtime Routines}

The interview's mealtime routine session provided insight to answer the following research question: how do parents of children with PFD view their roles when managing stressors during mealtime routines? A theme that developed from parental responses is that parents of children with PFD reported taking time outside of mealtimes to engage in activities 
that help reduce stress. During mealtimes, parents say too many things they must worry about and cannot be available for themselves.

Five out of five parents responded "yes" when asked if they have found the stressrelieving strategies helpful. However, they also reported not having enough time daily to engage in activities that alleviate stress. The most common response was to go outdoors to take a walk, go to the park, or go for a run. Another typical response was to talk with family and friends about stressful situations. The main challenge when implementing these strategies is time. Parents report not having sufficient time every single day to engage in their preferred activities to reduce stress. One parent reported that when he is unable to get outdoors, and he is having a stressful time during mealtime, he tends to "Walk away" from the stressful situation to avoid getting more upset. All parents, however, noted that they found their strategies used to reduce stress are helpful. One parent stated, "yes, a lot, absolutely. The one I use the most is going out to exercise/walk outside. I have also tried incorporating him into my walks." Figure 3 represents the methods parents use to reduce stress.

\section{Figure 3}

Methods to Reduce Stress 


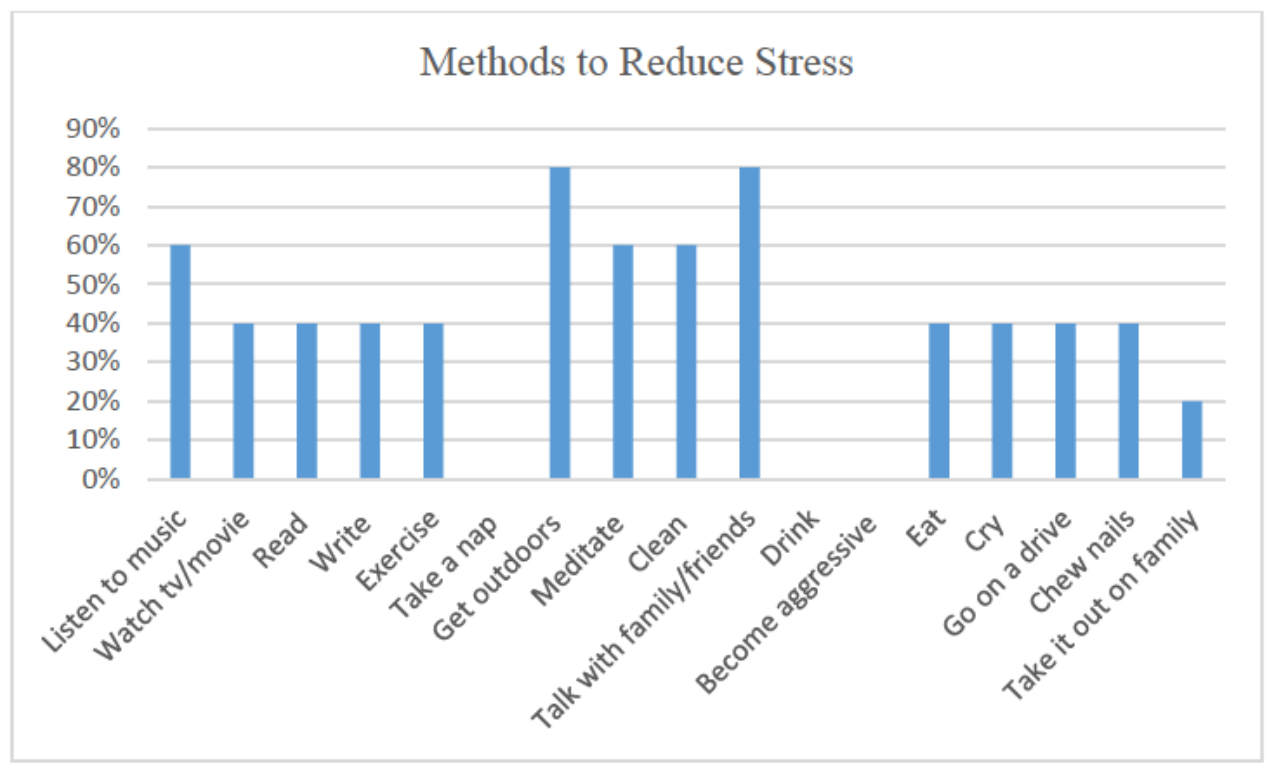

\section{Evidence of trustworthiness}

There are four components of trustworthiness in a qualitative research project. These components are credibility, transferability, dependability, and confirmability. Based on the information provided by the participants, all information is true and accurate, which are factors that lead to credibility. One of the project assumptions that was considered was that the participants in the study would answer the interview questions truthfully. To minimize this, parents were informed that the interviews were anonymous, and any information that could potentially identify the participant will not be published. When discussing the research findings, the student investigator compared the participants' information with research articles. The data was also discussed with the site supervisor and mentor, who are experts in pediatric feeding disorders and feeding therapy.

The current qualitative research can adjust to transferability because the study can apply to other populations, contexts, and situations. The student investigator provided participants' sociodemographic information, additional descriptive information, sample size, sample strategy, interview procedure, and inclusion and exclusion criteria. The information enables the reader to 
determine if this qualitative research study will be transferable or applicable to their setting. The student investigator provides an outline of the interview questionnaire and a time log breaking down weekly objectives in the appendices. Because this study was looking to gather parental perception of mealtime behaviors and parental stress, the findings can be applied to other studies to gather information regarding participant perception about a specific topic.

Dependability can refer to how the student investigator used consistency strategies throughout the study. In this case, the research records are maintained within an encrypted folder within the student investigator's personal computer. Access to this information is limited to the student investigator. The audio recordings are anonymized and stored within the encrypted folders and the data will be destroyed after three years. The student investigator and the mentor created the interview questionnaire. Before the administration of the interview questionnaire, the student investigator and the project mentor had a virtual meeting to discuss, review, and examine all aspects of the interview and data collection. The data collected from the interviews were discussed with the project mentor via zoom meeting. No information that could potentially identify the participant was discussed with the project mentor. The interviews' information and data were discussed with the site supervisor and other therapists on-site at a hospital in a southern state involved in the care of the children whose parents participated in the study; no participant identifiers were discussed.

The student investigator followed the necessary steps to ensure that the data is represented accurately. The information provided by the participants was transcribed verbatim to ensure accuracy and neutrality. The student investigator performed the transcription of the data. The interpretation of the data was based on the interview transcripts. The interview transcripts are stored within an encrypted folder within the student investigator's computer as well as in the 
form of excerpts on the database Dedoose. Throughout the results and analysis chapter, the reader can read exact quotes taken from the transcription of the interview questionnaire. This capstone project followed qualitative study regulations throughout.

\section{Conclusion}

The data collected during the parental interviews allowed the student investigator to answer the following questions: (1) how do parents of children with PFD perceive maladaptive behaviors during mealtime? (2) how do parents of children with PFD describe strategies to manage maladaptive mealtime behaviors during mealtime? And (3) how do parents of children with PFD view their roles when managing stressors during mealtime? The in-depth interviews provided an insight into the mealtime routines, mealtime behaviors, and parental stress for families of children with PFD. The student investigator was able to derive themes from the parental responses. All the themes and other pertinent information, including excerpts from the interviews, are described in this chapter to provide the reader with the parent's perception regarding mealtime routines, behaviors, and parental stress.

The learning objectives provided the student investigator with information about the multidisciplinary interventions being implemented for children with PFD, how a families' characteristics such as socioeconomic status can impact the implementation of strategies in the home environment, and the advantages and disadvantages of the current strategies used by parents to manage mealtime behaviors and parental stress. The objectives were met by creating weekly reflection papers based on information gathered during observation hours. The learning and project objectives, along with the research questions, contributed to solving the problem because they provided an insight into how parents manage behaviors and stress during mealtime.

Overall, the most frequently occurring mealtime behaviors are laughing, smiling, keeping 
mouth shut, using hands/arms to push food away, and eating too slow or too fast. During mealtime routines, four out of five $(80 \%)$ parents reported experiencing increased stress during mealtime routines averaging 7.4 on a scale from 1 to 10 . Overall, parents of children with PFD experience stress daily, averaging 6.8 on a scale from 1 to 10 . Parents' most common strategy to relieve stress is going outdoors and talking to family or friends. Although parents reported not having enough time daily to engage in stress-reducing strategy. Other parents also engage in activities such as meditation, cleaning, and listening to music. Based on the qualitative research study's current results, this study has the implications for filling the gap in research regarding how OTs involved in the care of children with PFD can assist parents in creating a positive mealtime environment that will improve mealtime behaviors and parental stress. 


\section{Chapter V: Summary, Conclusions, and Discussion}

\section{Introduction}

The capstone project aimed to identify and analyze how parents of children with pediatric feeding disorders manage maladaptive behaviors and stress during mealtime routines. Gaps in the research were identified, and it was found that the problem is that few research studies show how parents manage behaviors and stress during mealtime routines. The study participants completed an in-depth and in-person interview where they answered questions about mealtime routines, mealtime behaviors, and parental stress. This project was pursued because the student investigator wanted to fill the gap regarding parents' experiences during mealtime routines and how occupational therapists can assist in creating a positive mealtime environment that will benefit both the child and the family overall. Mealtime routines are influenced by physical, psychosocial, environmental, and social factors. The author believes that OTs have the expertise to improve mealtime routines for families of children with pediatric feeding disorders.

The qualitative research study was completed over a period of sixteen weeks including eleven weeks of observations of feeding therapy sessions. The student investigator completed the in-person interviews with the five participants at a hospital in a southern state. The interview questionnaire consisted of three sections: mealtime routines, mealtime behaviors, and parental stress. Parents reported that it was essential to create consistency during mealtimes by setting a mealtime routine. Sixty percent of parents reported that it takes around thirty to fortyfive minutes to complete a preferred meal. Five out of five parents reported that they must make separate meals for the child with PFD. As far as mealtime behaviors, five out of five parents reported that when their child is presented with non-preferred foods, the child pushes 
away the food. Three out of five reported that their child had behavior issues during mealtimes. The most frequently occurring mealtime behaviors are laughing and smiling. During the parental stress section, it was learned that three out of five parents report enjoying mealtimes with their children. Five out of five parents report feeling increased stress during mealtime routines. The average stress during mealtime is 7.4 on a scale from 1 to ten, compared to 6.8 for daily stress. The following section will outline the interpretation of the results of the current qualitative study.

\section{Interpretation of Results}

The findings from the current qualitative study confirm the knowledge from the literature review that parents of children with PFD experience increased stress during mealtime routines. This study answered the following research questions: (1) how do parents of children with PFD perceive maladaptive behaviors during mealtime? (2) how do parents of children with PFD describe strategies to manage maladaptive mealtime behaviors during mealtime? And (3) how do parents of children with PFD view their roles when managing stressors during mealtime?

\section{Research Question One}

The focus of research question one was how parents of children with PFD perceive maladaptive mealtime behaviors. The student investigator hypothesized that parents perceive mealtime as more challenging due to the presence of maladaptive behaviors. During the literature review, it was found that mealtime behaviors such as tantrums, aggression, headturning, throwing, and spitting food led parents to engage and create negative feeding practices. The negative feeding practices can lead to a negative mealtime environment and mealtime behaviors closely related to parental stress during mealtimes (Goodman et al., 2020; Murphy et 
al., 2020; Silverman et al., 2020). Silverman et al. (2020) also found that a child's level of aggression and emotional regulation is closely related to caregiver stress. The theme that was developed from this research question was that parents of children with PFD perceive mealtime behaviors as highly stressful. The theme was identified from questions one, five, and fourteen in the parental stress section. Five out of five parents reported feeling increased stress during mealtimes. The average mealtime stress was 7.4 on a scale from one to ten.

\section{Research Question Two}

The focus of research question two was about how parents of children with PFD describe strategies to manage maladaptive mealtime behaviors. The student investigator hypothesized that parents use positive or negative reinforcement, punishment, or rewards to manage behaviors during mealtime. During the literature review, it was found that mediating roles, communication skills, and reinforcement approaches can positively impact mealtime routines (Estrem et al., 2017). Structure mealtimes and positive interactions between parent and child can decrease conflict during mealtimes. A theme that developed with this research question was that parents of children with PFD reported ignoring maladaptive behaviors during mealtime. Five out of five parents reported that they wait for the behavior to stop. Parents also reported that when ignoring the behavior does not work, some parents soothe the child, and some use punishment such as taking away electronic devices.

\section{Research Question Three}

The focus of research question three was how parents of children with PFD view their roles when managing stressors during mealtimes. The student investigator hypothesized that parents view their daily roles as overwhelming when dealing with stress during mealtimes. While conducting the literature review, the student investigator found that mealtimes are highly 
influenced by parent feeding practices and styles, parental characteristics, and co-parenting situations. Parental feeding practices can be affected by anxiety and stress regarding mealtimes which can inadvertently impact behaviors during mealtime routines. The student investigator also hypothesized that parents would describe managing stress in relation to personal time rather than during mealtime routines, which means that parents will wait until after mealtime routines to take time for themselves rather than during mealtimes. While conducting the literature review, the student investigator found that parents of children with PFD spend an increased amount of time preparing foods, managing behaviors, and feeding the child. This does not leave time to manage stress during mealtimes. Parents that participated in the study reported getting outdoors and talking to family or friends as the primary way of reducing stress. Both of which occur outside of mealtime routines.

The literature review provided an insight into how a pediatric feeding disorder can affect mealtime routines, mealtime behaviors, and parental stress. Mealtime routines are essential to how family members interact, create memories, and create habits and routines. When there is a disruption in mealtime routines and parents cannot provide the family with structure, mealtimes get increasingly stressful and demanding for family members. Establishing a consistent mealtime routine is essential for family cohesion because it enables them to engage in positive mealtime experiences. Although mealtime routines are essential, only three out of five parents were able to have consistent daily mealtime routines. Two out of the five parents were unable to keep the same routines daily because of work schedules, coparenting arrangements, or therapy times. Other barriers to mealtimes include the environment and distractions such as tv or smartphones. Three out of four parents report that during mealtimes, the only distraction is talking amongst family members. Two out of four parents 
report using smartphones and tv during mealtime as distractions. The information regarding mealtime routines and distractions aligns with what was learned from literature. Just as distractions and routines can impact mealtime routines, mealtime behaviors can also cause a significant impact on mealtime routines.

Mealtime behaviors are highly prevalent in this population, and it was one of the main sections of the interview questionnaire. The literature review provided information about how parents' negative feeding practices or styles can affect mealtime behaviors because it leads parents to be more intrusive and controlling during mealtimes. On the contrary, a positive and supportive mealtime environment will create an experience that is more beneficial for the child and family. During the interviews, it was learned that to make mealtime more enjoyable, parents use toys, assorted flavors, incorporate the child in meal preparation, setting the table, and distraction to keep the child engaged and happy. Two parents also reported that it was very hard to make mealtimes enjoyable and that all they could do was feed the child the preferred foods so the child could get the calories he or she needs. Although there are many things that parents can do to create positive mealtimes and decrease behaviors when the strategies implemented are not effective, it leads the parents to experience increased stress during mealtime routines.

Stress during mealtime affects not only the parent but also the child and other family members. During the literature review, it was found that stress can affect the child's aggression and emotional regulation. The interview gave an insight into what parents do to manage stress and how this impacts the way parents engage with their child during mealtimes. During the interview, parents reported that if they are experiencing stress due to mealtime behaviors, they avoid behaviors, and sometimes they must walk away to avoid getting more frustrated. Parents 
engage in activities such as going outdoors, whether for walks or to the park, talking to family and friends. Parents also report talking to their child's therapists to get feedback or recommendations about what to do.

Throughout this entire process, from literature review to completing the capstone experience, the student investigator has learned that occupational therapy plays a significant role in the life of children with PFD and their families. During the capstone experience, the student investigator was fortunate to experience first-hand how OTs treat PFD and how they provide caregiver training and support. Besides providing treatments that focus on oral intake, oral motor skills, meal interventions, and mealtime behaviors management, the OT at the hospital was also in charge of providing weekly parent-training sessions. The parents of children in the intensive feeding program had weekly thirty-minute meetings where they could discuss the child's progress, ask questions about certain concerns they may have. The OT also took time to teach and demonstrate the strategies that were being used during therapy. During the last three weeks of the program, parents were present during feeding therapy sessions, and they oversaw implementing feeding strategies while receiving feedback from the therapist to ensure carry-over to the home environment.

The parent training and support that was received during the intensive feeding program showed an impact on the mealtime routines because five out of five parents reported being able to implement the clinic's strategies into the home environment. Although parents found the training helpful, they still have challenges implementing some strategies due to time, tantrums, push-back from the child, and co-parenting schedules. Only parents of children in the intensive feeding program receive this type of parent training. The children that undergo regular weekly feeding therapy at this facility do not receive the same parent training or support. Although 
therapists constantly update the parents regarding the strategies being used, the parents are not present during therapy sessions and do not have weekly meetings with the therapist. One parent whose child attends regular outpatient feeding therapy reported that although she tries to implement some strategies into the home environment, she has gotten to a point where she wants her child to eat, so it is more difficult. These results show that although parents implement strategies in the home environment, parents notice a difference when they are educated on the strategies.

The project, interview questionnaire, and capstone experience were guided by the theoretical models identified in chapters one and two. The student investigator used the EHP and PEOP models to address aspects of mealtime routines. The EHP model was incorporated in the questionnaire by addressing questions regarding cultural, physical, and social environments. The PEOP model was also integrated into the interview questionnaire by addressing aspects of mealtime relating to parents' intrinsic factors such as physiological, cognitive, psychological, and spiritual factors as well as extrinsic factors relating to cultural, societal factors, and social and economic systems. Questions also addressed parents' feelings regarding their parenting role and how they feel about their performance during mealtime routines. Specific questions are: Are any meals on the day family meals? During mealtimes, are there other activities going on in the area when your child is eating? Does your child eat the same or different across settings? How do you plan mealtimes so that they are enjoyable? Do you enjoy mealtimes with your child? When I have problems/difficulties with the care of my child, I seek help or advice. Do you feel overwhelmed in the parenting role? And do you find it difficult to balance the parenting role with other responsibilities? The general information questions or sociodemographic questions were also aligned to get an insight into how these 
characteristics, such as parents' employment, number of children, and people in the household, can affect mealtime routines.

Overall, occupational therapists play a significant role in the treatment of children with PFD and educate parents on strategies that can be implemented at home to help decrease mealtime behaviors and parental stress. During the capstone experience and observation hours, the student investigator witnessed how essential parent training can be and how in a short amount of time, it can make a difference in families' lives. It is essential to note that parent training sessions are not available to all parents, and some parents do not have the privilege to spend the thirty-minute weekly meetings. Making it much more critical for occupational therapists to incorporate these training sessions into their treatments. OTs can incorporate the families' unique cultural and social norms into the parent training to ensure that they can carry over. When parents were asked which support would be more beneficial to address mealtime behaviors and parental stress, five out of five parents responded that they would choose a parent-training course. Parents reported that they found the parent-training courses beneficial because they incorporate hands-on training.

\section{Strengths and Limitations}

The current project had several strengths. One strength is that the interview provided insight into parents' perspectives of how maladaptive behaviors and parental stress can impact mealtime routines. Parents identified the most frequently occurring mealtime behaviors and methods in which they decrease stress. A significant strength that allowed the student investigator to create stability with data was recording and transcribing data. The transcription of the interview assisted with the accuracy of data. It allowed the student investigator to use specific excerpts from the interview to give the reader insight into the parents' experiences about 
mealtime routines, mealtime behaviors, and parental stress. Another project strength is that the student investigator was present during parental training sessions and was able to experience first-hand the techniques that the OT is teaching the parents to enhance mealtime routines.

The project has several limitations: the five participants who participated in the study were recruited from a hospital in a southern state. Three of these participants were parents of children in the intensive feeding program. The intensive feeding program offers parent training and support sessions. The parents of children attending outpatient feeding therapy at the hospital do not get them, which creates a disadvantage. Another limitation is that the sample size is too small to be generalized to the entire population of parents of children with PFD. Although the research questions for this study were answered, they were all based on the literature review that the student investigator completed and can be biased, creating another limitation in this current research study. Lastly, the interview was only offered in English. With the large population of Spanish-speakers in South Florida, it would have been beneficial to offer the interview questionnaire in Spanish to allow more individuals to participate. The ongoing COVID-19 pandemic was also a limitation because only one parent was allowed in the waiting room, limiting parents' access.

\section{Recommendations for the Future}

Recommendations for future projects include developing and carrying out qualitative research studies of parents of children diagnosed with PFD who are undergoing general outpatient therapy. This will address the limitation of having participants whose children are undergoing feeding therapy in the intensive feeding program at a hospital in a southern state. Recruitment should also include parents of children undergoing feeding therapy at multiple outpatient clinics. That way, the investigator can compare the techniques used at different 
clinics. Future research studies should also include the interviews or questionnaires available in different languages that way, and it will be available to more participants. Although the student investigator used two different theoretical models to support the project, exploring other theoretical models will show other aspects of how occupational therapy can support this population.

Aside from research studies, future projects can also include project development by applying and generalizing this study's findings. This can be accomplished by testing out mealtime behavior reduction techniques and parental stress reduction techniques into a preintervention and post-intervention study. The parental strategies described in the current research study can be incorporated with other occupational therapy parent and family-based interventions that reinforce the importance of carry-over strategies. Future directions should also focus on OT practitioners advocating for parents of children with a PFD diagnosis because parents will have more opportunities to find strategies that will enhance the mealtime experience for everyone involved. Future directions should also focus on incorporating aspects of mealtime routines such as cultural, social, and environmental factors into the intervention process.

\section{Conclusions}

In conclusion, this paper supports how occupational therapists can become more involved in creating strategies that will assist parents of children with PFD in creating positive mealtime experiences by reducing mealtime behaviors and parental stress. Occupational therapists have the expertise and knowledge to assist parents by incorporating all aspects of mealtime routines, including rituals, routines, family dynamics, and environmental and social factors. Mealtimes have shown to provide excellent benefits for the child, such as healthier eating habits, improved 
behavior, better academic performance, and the more resources that parents have available, the more chances they must create positive mealtime experiences (Jones, 2018). This paper supports that with more resources such as parent-training, parents will improve the carry-over of techniques learned in the clinic into the home environment, improving the child's progress in therapy. 


\section{References}

American Occupational Therapy Association. (2014). Occupational therapy practice framework: Domain and process ( $3^{\text {rd }}$ ed.). American Journal of Occupational Therapy, 68(Suppl. 1), S1-S48. http://dx.doi.org/10.5012/ajot.2014.682006

American Occupational Therapy Association. (2017). The practice of occupational therapy in feeding, eating, and swallowing. American Journal of Occupational Therapy, 71(Suppl. 2), 7112410015. https://doi.org/10.5014/ ajot.2017.716S04

American Psychiatric Association, DSM 5 Task Force. (2013). DSM-5: Diagnostic and statistical manual of mental disorders (5th ed.). Arlington, VA: American Psychiatric Publishing.

Ausderau, K., \& Juarez, M. (2013). The impact of autism spectrum disorders and eating challenges on family mealtimes. ICAN: Infant, Child, \& Adolescent Nutrition, 5(5), 315323. https://doi.org/10.1177/1941406413502808

Ausderau, K. K., St. John, B., Kwaterski, K. N., Nieuwenhuis, B., \& Bradley, E. (2019). Parents' strategies to support mealtime participation of their children with autism spectrum disorder. American Journal of Occupational Therapy, 73(1), 1-10. https://doi.org/10.5014/ajot.2019.024612

Aviram, I., Atzaba-Poria, N., Pike, A., Meiri, G., \& Yerushalmi, B. (2015). Mealtime dynamics in child feeding disorder: the role of child temperament, parental sense of competence, and paternal involvement. Journal of pediatric psychology, 40(1), 45-54. https://doi.org/10.1093/jpepsy/jsu095 
Bahr, D., \& Johanson, N. (2013). A family-centered approach to feeding disorders in children (birth to 5-years). Perspectives on swallowing and swallowing disorders (dysphagia), 22(4), 161-171. https://doi.org/10.1044/sasd22.4.161

Baum, C. M., \& Christiansen, C. H. (2005). Person-environment-occupation-performance: An occupation-based framework for practice. In C. H. Christiansen, C. M. Baum, \& J. BassHaugen (Eds.), Occupational therapy: Performance, 47 participation, and well-being (3rd ed.). (pp. 243-257). Thorofare, NJ: SLACK Inc.

Baumrind, D. (1991). Effective parenting during the early adolescent transition. In P. A. Cowan \& E. M. Hetherington (Eds.), Family transitions. (pp. 111-163). Lawrence Erlbaum Associates, Inc.

Bearss, K., Johnson, C., Smith, T., Lecavalier, L., Swiezy, N., Aman, M., McAdam, D. B., Butter, E., Stillitano, C., Minshawi, N., Sukhodolsky, D. G., Mruzek, D. W., Turner, K., Neal, T., Hallett, V., Mulick, J. A., Green, B., Handen, B., Deng, Y., Dziura, J., ... Scahill, L. (2015). Effect of parent training vs parent education on behavioral problems in children with autism spectrum disorder: a randomized clinical trial. JAMA, 313(15), 1524-1533. https://doi.org/10.1001/jama.2015.3150

Berlin, K. S., Davies, W. H., Silverman, A. H., \& Rudolph, C. D. (2011). Assessing familybased feeding strategies, strengths, and mealtime structure with the Feeding Strategies Questionnaire. Journal of pediatric psychology, 36(5), 586-595. https://doi.org/10.1093/jpepsy/jsp107

Berry, JD, \& Jones, W, H, (1995) The Parental Stress Scale: initial psychometric evidence. Journal of Social and Personal Relationships, 12, 463 - 472. 
Bonds, S. (n.d). Understanding families: family dynamics [fact sheet]. Retrieve from http://www.strongbonds.jss.org.au/workers/families/dynamics.html

Borowitz, K. C., \& Borowitz, S. M. (2018). Feeding Problems in Infants and Children: Assessment and Etiology. Pediatric clinics of North America, 65(1), 59-72. https://doi.org/10.1016/j.pcl.2017.08.021

Coldiron, Michael A., The experiences of the parents of children undergoing occupational therapy intervention for the occupation of feeding (2016). Occupational Therapy Doctorate Capstone Projects. 9.

Cole, M. B., \& Tufano, R. (2008). Applied theories in occupational therapy: a practical approach. Thorofare, NJ.: Slack.

Curtin, C., Hubbard, K., Anderson, S., Mick, E., Must, A., \& Bandini, L. (2015). Food selectivity, mealtime behavior problems, spousal stress, and family food choices in children with and without autism spectrum disorder. Journal of Autism \& Developmental Disorders, 45(10), 3308-3315. https://doi.org/10.1007/s10803-015-2490-x

Curtiss, S. L., \& Ebata, A. T. (2019). The nature of family meals: A new vision of families of children with autism. Journal of Autism \& Developmental Disorders, 49(2), 441-452. https://doi.org/10.1007/s10803-018-3720-9

Creswell, J. W., \& Creswell, J. D. (2018). Research design: Qualitative, quantitative, and mixed methods approach. Los Angeles: Sage Publications.

Crowe, T. K., Freeze, B., Provost, E., King, L., \& Sanders, M. (2016). Maternal perceptions of nutrition, stress, time, and assistance during mealtimes: similarities and differences between mothers of children with autism spectrum disorders and mothers of children with 
typical development. Journal of Occupational Therapy, Schools \& Early Intervention, 9(3), 242-257.

Didehbani, N. (2009). Role of parental anxiety on pediatric disorders. University of North Texas.

Didehbani, N., Kelly, K., Austin, L., \& Wiechmann, A. (2011). Role of parental stress on pediatric feeding disorders. Childrens Health Care, 40(2), 85-100. https://doi.org/10.1080/02739615.2011.564557

Estrem, H. H., Pados, B. F., Thoyre, S., Knafl, K., McComish, C., \& Park, J. (2016). Concept of pediatric feeding problems from the parent perspective. MCN: The American Journal of Maternal Child Nursing, 41(4), 212-220.

Estrem, H. H., Pados, B. F., Park, J., Knafl, K. A., \& Thoyre, S. M. (2017). Feeding problems in infancy and early childhood: evolutionary concept analysis. Journal of Advanced Nursing (John Wiley \& Sons, Inc.), 73(1), 56-70. https://doi.org/10.1111/jan.13140

Estrem, H. H., Thoyre, S. M., Knafl, K. A., Frisk Pados, B., \& Van Riper, M. (2018). "It's a long-term process": description of daily family life when a child has a feeding disorder. Journal of pediatric health care: official publication of National Association of Pediatric Nurse Associates \& Practitioners, 32(4), 340-347.

https://doi.org/10.1016/j.pedhc.2017.12.002

Fiese, B. H., Foley, K. P., \& Spagnola, M. (2006). Routine and ritual elements in family mealtimes: contexts for child well-being and family identity. New Directions for Child and Adolescent Development, 111, 67-89.

Fishbein, M., Benton, K., \& Struthers, W. (2016). Mealtime disruption and caregiver stress in referrals to an outpatient feeding clinic. JPEN Journal of Parenteral \& Enteral Nutrition, 40(5), 636-645. https://doi.org/10.1177/0148607114543832 
Gedney, L. (2013). Family mealtime. Today's Dietitian, 15(10), 30-36.

Goday, P. S., Huh, S. Y., Silverman, A., Lukens, C. T., Dodrill, P., Cohen, S. S., Delaney, A. L., Feuling, M. B., Noel, R. J., Gisel, E., Kenzer, A., Kessler, D. B., Kraus de Camargo, O., Browne, J., \& Phalen, J. A. (2019). Pediatric feeding disorder: Consensus definition and conceptual framework. Journal of pediatric gastroenterology and nutrition, 68(1), 124129. https://doi.org/10.1097/MPG.0000000000002188

González, M. L., \& Stern, K. (2016). Co-occurring behavioral difficulties in children with severe feeding problems: A descriptive study. Research in Developmental Disabilities, 58, 4554. https://doi.org/10.1016/j.ridd.2016.08.009

Goodman, L. C., Roberts, L. T., \& Musher-Eizenman, D. R. (2020). Mindful feeding: A pathway between parenting style and child eating behaviors. Eating behaviors, 36, 101335. https://doi.org/10.1016/j.eatbeh.2019.101335

Greer, A. J., Gulotta, C. S., Masler, E. A., \& Laud, R. B. (2008). Caregiver stress and outcomes of children with pediatric feeding disorders treated in an intensive interdisciplinary program. Journal of Pediatric Psychology, 33(6), 612-620. https://doi.org/10.1093/jpepsy/jsm116

Gueron-Sela, N., Atzaba-Poria, N., Meiri, G., \& Yerushalmi, B. (2011). Maternal worries about child underweight mediate and moderate the relationship between child feeding disorders and mother-child feeding interactions. Journal of pediatric psychology, 36(7), 827-836. https://doi.org/10.1093/jpepsy/jsr001

Harris, H. A., Jansen, E., \& Rossi, T. (2020). 'It's not worth the fight': Fathers' perceptions of family mealtime interactions, feeding practices and child eating behaviours. Appetite, 150, 104642. https://doi.org/10.1016/j.appet.2020.104642 
Hinojosa, J., Kramer, P., Royeen, C. B., \& Dunn, W. (2017). In Perspectives on Human Occupation: theories underlying practice (pp. 207-235). essay, F.A. Davis Company.

Howe, T., \& Wang, T. (2013). Systematic Review of Interventions Used in or Relevant to Occupational Therapy for Children with feeding difficulties ages birth-5 Years. American Journal of Occupational Therapy, 67(4), 405-412. https://doi.org/10.5014/ajot.2013.004564

Johnson, C. R., Brown, K., Hyman, S. L., Brooks, M. M., Aponte, C., Levato, L., Schmidt, B., Evans, V., Huo, Z., Bendixen, R., Eng, H., Sax, T., \& Smith, T. (2019). Parent training for feeding problems in children with autism spectrum disorder: Initial randomized trial. Journal of Pediatric Psychology, 44(2), 164-175. https://doi.org/10.1093/jpepsy/jsy063

Johnson, C. R., Foldes, E.sDeMand, A., \& Brooks, M. M. (2015). Behavioral parent training to address feeding problems in children with autism spectrum disorder: A pilot trial. Journal of Developmental and Physical Disabilities, 27(5), 591-607. https://doi.org/10.1007/s10882-015-9437-1

Jones, B. L. (2018). Making time for family meals: Parental influences, home eating environments, barriers and protective factors. Physiology \& Behavior, 193, 248-251. https://doi.org/10.1016/j.physbeh.2018.03.035

Kramer, S. (2020, May 30). U.S. has world's highest rate of children living in single-parent households. Retrieved from https://www.pewresearch.org/fact-tank/2019/12/12/u-schildren-more-likely-than-children-in-other-countries-to-live-with-just-one-parent/ 
Ledford, J. R., Whiteside, E., \& Severini, K. E. (2018). A systematic review of interventions for feeding-related behaviors for individuals with autism spectrum disorders. Research in Autism Spectrum Disorders, 52, 69-80. https://doi.org/10.1016/j.rasd.2018.04.008

Ledford, J. R., \& Gast, D. L. (2006). Feeding problems in children with autism spectrum disorders: a review. focus on autism and other developmental disabilities, 21(3), 153166. https://doi.org/10.1177/10883576060210030401

Marshall, J., Hill, R. J., Ziviani, J., \& Dodrill, P. (2014). Features of feeding difficulty in children with autism spectrum disorder. International Journal of Speech-Language Pathology, 16(2), 151-158. https://doi.org/10.3109/17549507.2013.808700

Marshall, J., Ware, R., Ziviani, J., Hill, R. J., \& Dodrill, P. (2015). Efficacy of interventions to improve feeding difficulties in children with autism spectrum disorders: a systematic review and meta-analysis. Child: Care, Health \& Development, 41(2), 278-302. https://doi.org/10.1111/cch.12157

Murphy, J., Zlomke, K., Vanormer, J., \& Swingle, H. (2020). Impact of disruptive behavior in childhood feeding difficulties. Journal of Clinical Psychology in Medical Settings, 27(2), 406-415. doi:10.1007/s10880-019-09646-y

Paul, S., \& D’Amico, M. (2013). The role of occupational therapy in the management of feeding and swallowing disorders. New Zealand Journal of Occupational Therapy, 62(2), 27 31.

Postorino, V., Sanges, V., Giovagnoli, G., Fatta, L. M., De Peppo, L., Armando, M., Vicari, S., \& Mazzone, L. (2015). Clinical differences in children with autism spectrum disorder with and without food selectivity. Appetite, 92, 126-132. https://doi.org/10.1016/j.appet.2015.05.016 
Rigal, N., Chabanet, C., Issanchou, S., \& Monnery-Patris, S. (2012). Links between maternal feeding practices and children's eating difficulties. Validation of French tools. Appetite, 58(2), 629-637. https://doi.org/10.1016/j.appet.2011.12.016

Sharp, W. G., Jaquess, D. L., Morton, J. F., \& Herzinger, C. V. (2010). Pediatric feeding disorders: a quantitative synthesis of treatment outcomes. Clinical child and family psychology review, 13(4), 348-365. https://doi.org/10.1007/s10567-010-0079-7

Sharp, W. G., Berry, R. C., McCracken, C., Nuhu, N. N., Marvel, E., Saulnier, C. A., Klin, A., Jones, W., \& Jaquess, D. L. (2013). Feeding problems and nutrient intake in children with autism spectrum disorders: a meta-analysis and comprehensive review of the literature. Journal of autism and developmental disorders, 43(9), 2159-2173. https://doi.org/10.1007/s10803-013-1771-5

Sharp, W. G., Volkert, V. M., Scahill, L., McCracken, C. E., \& McElhanon, B. (2017). A Systematic Review and Meta-Analysis of Intensive Multidisciplinary Intervention for Pediatric Feeding Disorders: How Standard Is the Standard of Care? The Journal of pediatrics, 181, 116-124.e4. https://doi.org/10.1016/j.jpeds.2016.10.002Simon, M. K. (2011). Dissertation and scholarly research: Recipes for success (2011 Ed.). Seattle, WA, Dissertation Success, LLC.

Spagnola, M., \& Fiese, B. H. (2007). Family routines and rituals: A context for development in the lives of young children. Infants \& Young Children, 20(4), 284-299. https://doi.org/10.1097/01.iyc.0000290352.32170.5a

Silverman, A. H., Erato, G., \& Goday, P. (2020). The relationship between chronic pediatric feeding disorders and caregiver stress. Journal of Child Health Care, 136749352090538. https://doi.org/10.1177/1367493520905381 
Suarez, M. A., Atchinson, B. J., \& Lagerwey, M. (2014). Brief report-phenomenological examination of the mealtime experience for mothers of children with autism and food selectivity. American Journal of Occupational Therapy, 68, 102-107. https://doi.org/10.5014/ajot.2014.008748

Thullen, M., \& Bonsall, A. (2017). Co-parenting quality, parenting stress, and feeding challenges in families with a child diagnosed with autism spectrum disorder. Journal of Autism \& Developmental Disorders, 47(3), 878-886. https://doi.org/10.1007/s10803-016-2988-X

Twachtman-Reilly J, Amaral SC, \& Zebrowski PP. (2008). Addressing feeding disorders in children on the autism spectrum in school-based settings: physiological and behavioral issues. Language, Speech \& Hearing Services in Schools, 39(2), 261-272.

Utter, J., Larson, N., Berge, J. M., Eisenberg, M. E., Fulkerson, J. A., \& Neumark-Sztainer, D. (2018). Family meals among parents: Associations with nutritional, social and emotional wellbeing. Preventive medicine, 113, 7-12. https://doi.org/10.1016/j.ypmed.2018.05.006

Yang, H. R. (2017). How to approach feeding difficulties in young children. Korean Journal of Pediatrics, 60(12), 379. https://doi.org/10.3345/kjp.2017.60.12.379 


\section{Appendices}

\section{Appendix A}

\section{Weekly Time Log}

\begin{tabular}{|c|c|c|c|c|c|}
\hline Week & Date & $\begin{array}{l}\text { Direct Hours } \\
\text { at a Hospital }\end{array}$ & Indirect Hours & $\begin{array}{l}\text { Learning } \\
\text { Objective }\end{array}$ & Specific \\
\hline 1 & $1 / 11-1 / 15$ & 30 & 10 & $\begin{array}{l}\text { L1, L7, } \\
\text { L10, L9, } \\
\text { PB9 }\end{array}$ & $\begin{array}{l}\text {-Observation of feeding } \\
\text { therapy sessions } \\
\text { - Gather information } \\
\text { about PFD through } \\
\text { webinars, videos, } \\
\text { research articles, and } \\
\text { conference material } \\
\text { provided by } \\
\text { mentor/facility } \\
\text {-Complete deliverable } \\
\text {-Weekly parent/therapist } \\
\text { meeting } \\
\text {-Weekly meeting with } \\
\text { mentor }\end{array}$ \\
\hline 2 & $1 / 18-1 / 22$ & 30 & 11 & $\begin{array}{l}\text { L1, L3, } \\
\text { L7, L8, } \\
\text { L9, PB9 }\end{array}$ & $\begin{array}{l}\text {-Observation of feeding } \\
\text { therapy } \\
\text { sessions/interdisciplinary } \\
\text { - Gather information } \\
\text { about PFD through } \\
\text { webinars, videos, } \\
\text { research articles, and } \\
\text { conference material } \\
\text { provided by } \\
\text { mentor/facility } \\
\text {-Ask parents about carry- } \\
\text { over techniques to the } \\
\text { home environment } \\
\text {-Complete deliverable } \\
\text {-Weekly parent/therapist } \\
\text { meeting } \\
\text {-Weekly meeting with } \\
\text { mentor }\end{array}$ \\
\hline
\end{tabular}


3

$1 / 25-1 / 29 \quad 40$

4

$2 / 8-2 / 12 \quad 30$

5
$2 / 1-2 / 5 \quad 30$
10

13

19
L1, L4, -Observation of feeding therapy sessions

L8, L10, - Gather information about PFD through

L9, PB9 webinars, videos, research articles, and conference material provided by mentor/facility -Ask parents how family characteristics affect carry over techniques -Complete deliverable -Weekly parent/therapist meeting

-Weekly meeting with mentor

L1, PB2, -Observation of feeding therapy sessions

L8, L10, -Interview parents

- Gather information

L9, PB8 about PFD through webinars, videos, research articles, and conference material provided by mentor/facility -Complete deliverable -Weekly parent/therapist meeting

-Weekly meeting with mentor

19

\section{L1, PB2, -Observation of feeding} therapy sessions

PB3, L8, -Interview parents -Transcribe data

L10, L9, - Gather information about PFD through

PB9 webinars, videos, research articles, and conference material provided by mentor/facility -Complete deliverable -Weekly parent/therapist meeting 


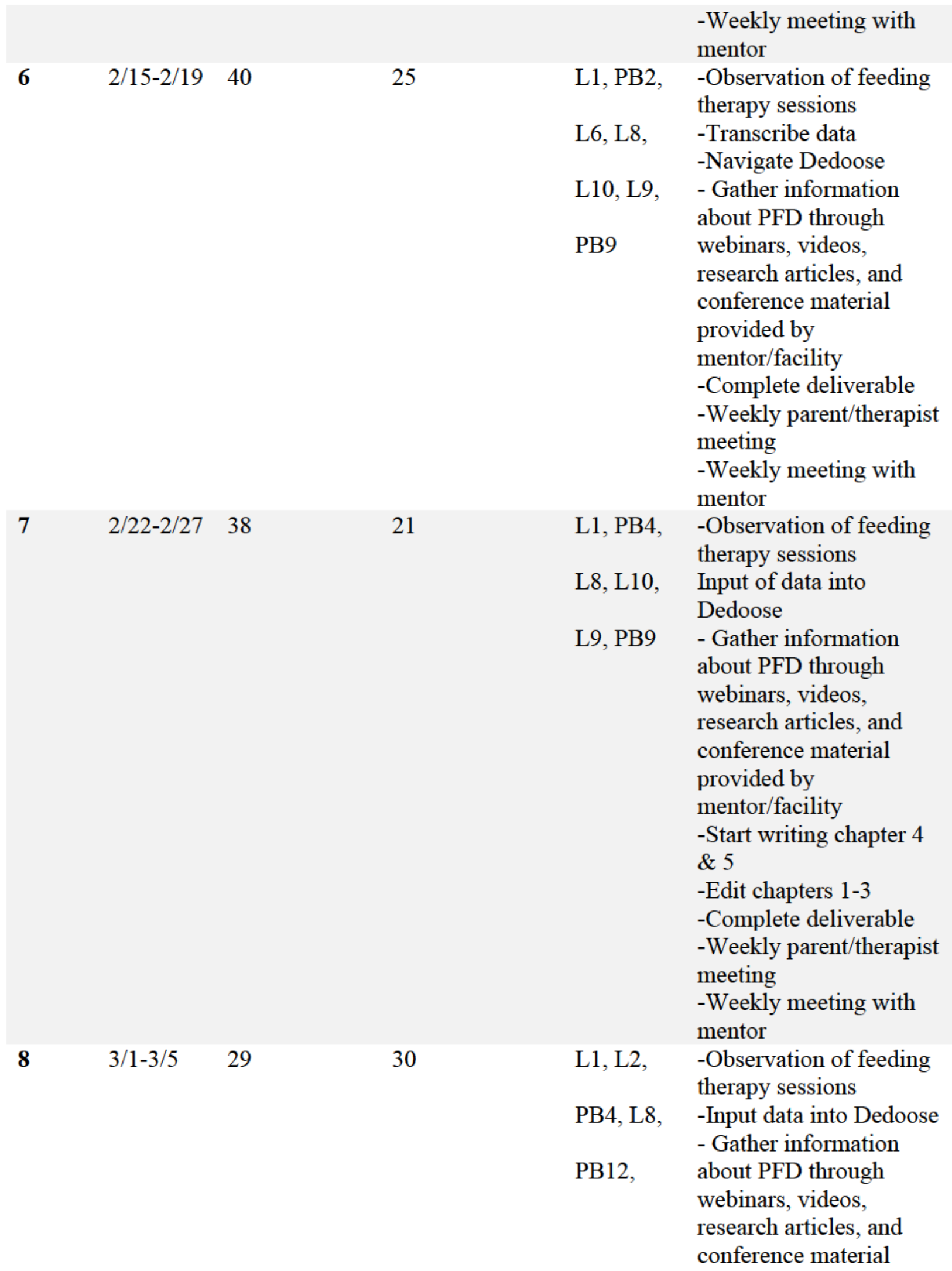




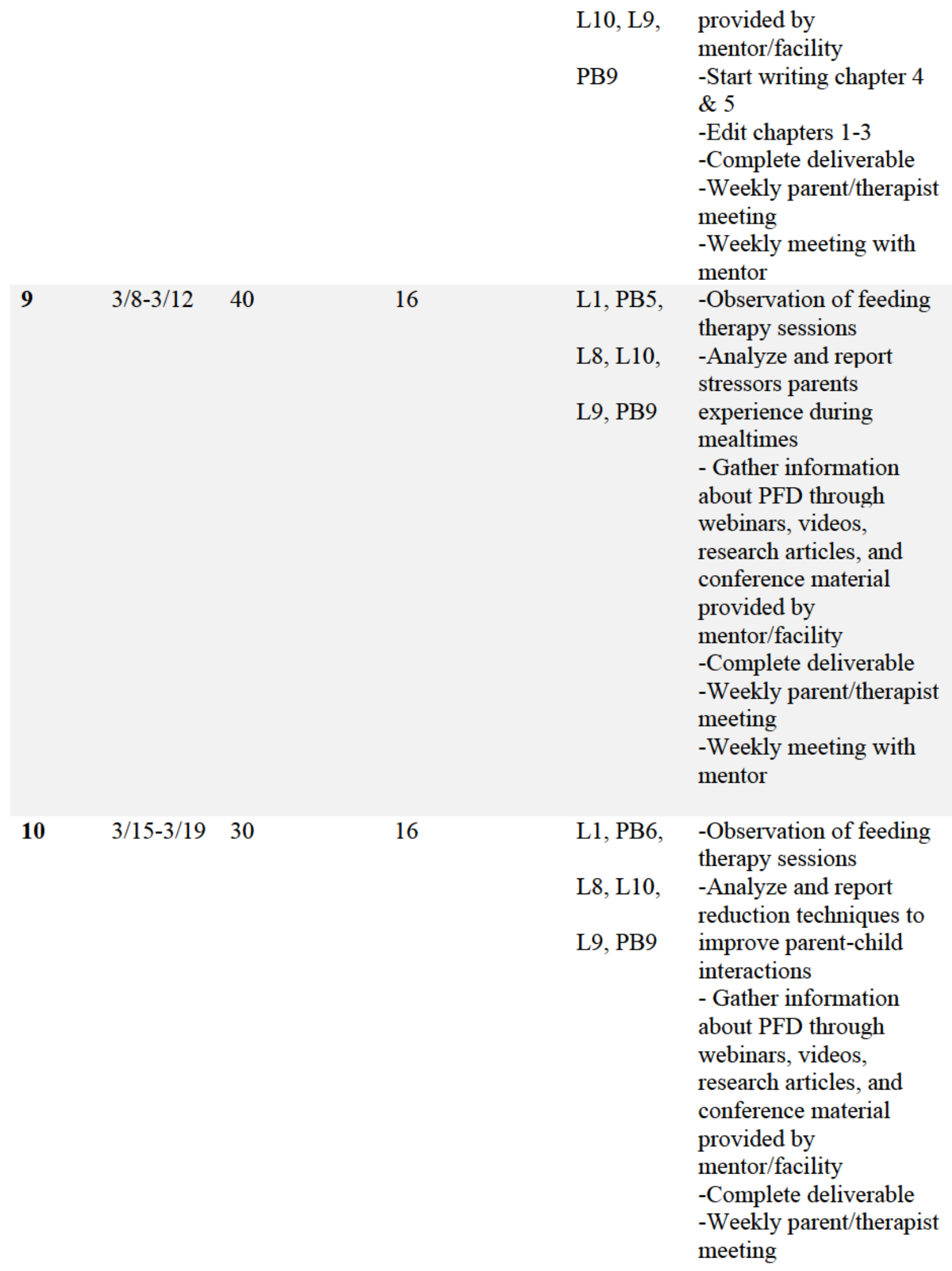


$113 / 22-3 / 26 \quad 40$

$123 / 29-4 / 2$

$13 \quad 4 / 5-4 / 9$

$14 \quad 4 / 12-4 / 16$
12

11

5

11
-Weekly meeting with mentor

PB7, L10 -Observation of feeding therapy sessions -Analyze and report most frequently occurring maladaptive mealtime behaviors and strategies used to manage behaviors

- Gather information about PFD through webinars, videos, research articles, and conference material provided by mentor/facility -Complete deliverable -Analyze an report similarities and differences of strategies used by families to

PB12 manage behaviors and stress

-Share findings with facility

-Chapter $4 \& 5$ revisions

PB12, -Chapter $4 \& 5$ revisions -Start creating poster

PB11

PB11, -Put chapters 1-5 together

PB12, -Create a poster for dissemination using

PB13, USA template -Submit completed

PB14 project -Submit paper to journals and feeding matters 


\title{
Appendix B
}

\section{Participant Information Sheet}

\section{Qualitative Exploration of Mealtime Routines, Behaviors, and Parental Stress of Children}

\author{
with Pediatric Feeding Disorders: Analysis of Occupational Performance
}

I would like to invite you to take part in a research study. Before you decide you need to understand why the research is being done and involvement you would have. Please take a moment to read the following information carefully. Ask questions if you need clarification or if you would like more information. Take time to decide whether or not you can take part in the study. You may contact Andrea Patino at a.patino@usa.edu with any questions.

\section{Who am I and what this study is about?}

My name is Andrea Patino and I am an occupational therapy doctorate student at the University of St. Augustine for Health Sciences (Miami Campus). I am conducting research in partial fulfillment of a Doctorate degree. The overall aim of the study is to identify strategies used to manage mealtime behaviors and parental stress for parents of children with pediatric feeding disorders

What will taking part involve?

Your participation in this research study will consist of answering interview questions regarding your family's mealtime routines. The anticipated duration of your participation is approximately one-hour, which will be dependent on participant response. You will be required to sign a consent form prior to their participation in this study. The ways we will protect your privacy and confidentiality are described in a separate section later in this form.

We will make an audio recording of the interview responses to ensure accuracy of data collection Why have you been invited to take part?

You have been invited to take part in this study because your child has diagnoses of pediatric feeding disorder.

Do you have to take part?

It is up to you to decide whether to take part in the study. You can withdraw at any time and you do not have to give a reason for withdrawal. If you decide to take part you will be given this information sheet to keep and will be asked to sign a consent form.

What are the possible risks and benefits of taking part?

You will receive no direct benefit from being in this study. Your being in this study may help the investigators learn different strategies and techniques that parents utilize to manage mealtime behaviors and parental stress. Contributing to the body of research may be identified as a benefit because it will assist occupational therapists and other professions in creating new parentdirected interventions to help reduce maladaptive mealtime behaviors and parental stress. Risks are no more than those encountered in everyday life. If at any point you feel uncomfortable answering a question, feel free to stop at any time. If you decide that you want to stop being in the study, we ask that you let us know.

\section{Will taking part be confidential?}

Your participation in this study will be strictly confidential. Any information that you provide that could be used to identify you will not be published. 
How will information you provide be recorded, stored, and protected?

Consent forms and original audio will be retained within an encrypted folder on the student investigator's personal computer. No information that could potentially identify you will be published. All data will be destroyed after 3 years following the completion of the study.

Who should you contact for further information?

Andrea Patino Occupational Therapy Doctorate Student at the University of St. Augustine for Health Sciences

Email:A.patino@usa.edu

THIS PROJECT HAS BEEN REVIEWED AND APPROVED BY THE UNIVERSITY OF ST. AUGUSTINE FOR HEALTH SCIENCES INSTITUTIONAL REVIEW BOARD FOR THE PROTECTION OF HUMAN SUBJECTS.

IF YOU HAVE QUESTIONS OR CONCERNS, THOSE QUESTIONS OR CONCERNS SHOULD BE DIRECTED TO THE INSTITUTIONAL IRB CHAIR, DR. ELIZABETH ARDOLINO, EMAIL: EARDOLINO@USA.EDU, PHONE: 737-202-3343 


\section{Appendix C}

\section{Informed Consent}

Principal Investigator:

Name: Dr. Pamela Kasyan-Howe, Doctoral Coordinator

Phone Number: 305-992-1410

Email: pkasyanhowe@usa.edu

Co-Investigator:

Name: Andrea Patino, Occupational Therapy Doctorate Student

Phone Number: 305-984-7215

Email: a.patino@usa.edu

You are being asked to participate in a research study. The box below highlights key information about this research for you to consider when making a decision whether or not to participate. Carefully consider this information and the more detailed information provided below the box. Please ask questions about any of the information you do not understand before you decide whether to participate.

Key Information for You to Consider

- Voluntary Consent: You are being asked to volunteer for a research study. It is up to you whether you choose to participate or not. There will be no penalty or loss of benefits to which you are otherwise entitled if you choose not to participate or discontinue participation.

- Purpose: The purpose of this research is to identify strategies used to manage maladaptive mealtime behaviors and parental stress for parents of children with pediatric feeding disorder and co-occurring autism spectrum disorder.

- Duration: It is expected that your participation will be one 60-minute interview session

- Procedures and Activities: You will be asked to answer questions regarding your mealtime routine, maladaptive mealtime behaviors, and parental stress.

- Risks: Risks are no more than those encountered in everyday life.

- Benefits: The participant will receive no personal benefits other than contributing to the body of research to further assist children with pediatric feeding disorders and co-occurring autism spectrum disorder

- Alternatives: Participation is voluntary, and the only alternative is to not participate.

\section{Why is this research being done?}

The overall aim of the study is to identify strategies used to manage maladaptive mealtime behaviors and parental stress for parents of children with pediatric feeding disorder and cooccurring autism spectrum disorder What will happen in this research study?

Your participation in this research study will consist of answering interview questions and an assessment regarding your family's mealtime routines. More specifically, you will be asked how 
you manage maladaptive behaviors and stress during mealtime routines. The anticipated duration of your participation is one-hour, which will be dependent on participant response. Individuals are required to sign this consent form prior to their participation in this study. The ways we will protect your privacy and confidentiality are described in a separate section later in this form. We will make an audio recording of the interview responses to ensure accuracy of data collection

\section{Risks and Discomforts}

Risks are no more than those encountered in everyday life. If at any point you feel uncomfortable answering a question, feel free to stop at any time. If you decide that you want to stop being in the study, we ask that you let us know.

\section{Potential Benefits}

You will receive no direct benefit from being in this study. Your being in this study may help the investigators learn different strategies and techniques that parents utilize to manage maladaptive mealtime behaviors and parental stress. Contributing to the body of research may be identified as a benefit because it will assist occupational therapists and other professions in creating new parent-directed interventions to help reduce maladaptive mealtime behaviors and parental stress.

\section{Costs}

There are no costs to you for being in this research study.

\section{Payment}

You will not be paid for being in this study.

\section{Confidentiality}

The research records will be maintained within an encrypted folder within the student investigator's personal computer. Access to the information will be limited to the student investigator (Andrea Patino), and the supervising investigator (Dr. Kasyan-Howe). The audio recordings will be anonymized and stored within an encrypted folder. With your permission I may include selective quotes from the transcription to illustrate points. No information that could potentially identify you will be published. All data will be destroyed after 3 years following the completion of the study.

\section{$\underline{\text { Re-Contact }}$}

this study
study

\section{Subject's Rights}

By consenting to be in this study you do not waive any of your legal rights. Consenting means that you have been given information about this study and that you agree to participate in the study. You will be given a copy of this form to keep.

If you do not agree to be in this study or if at any time you withdraw from this study, you will not suffer any penalty or lose any benefits to which you are entitled. Your participation is completely up to you. Your decision will not affect your ability to get health care or payment for your health care. It will not affect your enrollment in any health plan or benefits you can get.

\section{Questions}

The investigator or a member of the research team will try to answer all your questions. If you have questions or concerns at any time, contact Andrea Patino at (305)-984-7215

You may also call 737-202-3343 or email eardolino@usa.edu. You will be talking to Elizabeth Ardolino, the chairperson of the IRB (INSTITUTIONAL REVIEW BOARD) at the University 
of St. Augustine for Health Sciences. The IRB is a group that helps monitor research. You should call or email the IRB if you want to find out about your rights as a research subject. You should also call or email if you want to talk to someone who is not part of the study about your questions, concerns, or problems.

Subject:

Printed name of subject

By signing this consent form, you are indicating that

- you have read this form (or it has been read to you)

- your questions have been answered to your satisfaction

- you voluntarily agree to participate in this research study

Signature of subject

Date

\section{Researcher:}

Printed name of person conducting consent discussion

I have personally explained the research to the above-named subject and answered all questions. I believe that the subject understands what is involved in the study and freely agrees to participate.

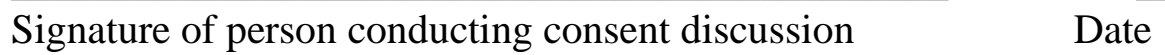

THIS PROJECT HAS BEEN REVIEWED AND APPROVED BY THE UNIVERSITY OF ST. AUGUSTINE FOR HEALTH SCIENCES INSTITUTIONAL REVIEW BOARD FOR THE PROTECTION OF HUMAN SUBJECTS.

IF YOU HAVE QUESTIONS OR CONCERNS, THOSE QUESTIONS OR CONCERNS

SHOULD BE DIRECTED TO THE INSTITUTIONAL IRB CHAIR, DR. ELIZABETH

ARDOLINO, EMAIL: EARDOLINO@USA.EDU, PHONE: 737-202-3343 


\section{Appendix D}

\section{Interview Questionnaire}

\section{General information}

1. What is your child's age?

2. What is your child's sex? Male Female

3. How many people live in your household?

4. Mother's age

5. Mother's employment

6. Father's age

7. Father's employment

8. Current diagnoses

9. Number of siblings

a. What order was your child born in relation to siblings: $1^{\text {st }} 2^{\text {nd }} 3^{\text {rd }} 4^{\text {th }} 5^{\text {th }}$ other:

10. What services is your child currently receiving: occupational therapy speech therapy feeding therapy

11. When was your child diagnosed with:

Feeding disorder

Autism spectrum disorder

\section{Mealtime Routine Section}

1. How many times a day does your child eat?

2. Do you think your child eats enough throughout the day?

3. Does your child eat at the same time every day?

Please explain

4. Does your child let you know when he/she is hungry?

Please explain

5. Do you have concerns regarding your child's weight?
a. Yes
b. No
c. Explain

6. Does your child self-feed?
a. Yes
b. No
c. What utensils are used?

i. Spoon

ii. Fork

iii. Spork

7. How long does it usually take for your child to eat a meal/to feed your child?
a. 5-30 minutes
b. $30-45$ minutes
c. More than an hour 
8. How is your child seated during mealtime? (i.e., regular chair, highchair, booster seat, standing, other)
a. Regular chair
b. Highchair
c. Booster seat
d. Standing

9. Are any meals in the day family meals?

10. Does your child eat the same food as the rest of the family?

11. What meal does your child do best with?
a. Breakfast
b. Lunch
c. Snacks
d. Dinner

12. During mealtimes, are there other activities going on in the area when your child is eating?
a. TV is on
b. Music is on
c. Phone activity
d. Talking amongst family members

13. Does your child eat the same or different across settings? (i.e., restaurants, school, friend/family's house)

\section{Mealtime Behavior Section}

1. Does your child have any behavior issues?

\section{Explain}

2. Does your child have any attention difficulties?

\section{Explain}

3. Does your child usually enjoy eating?

\section{Explain}

4. Does your child have difficulty with sensory processing? (i.e., noises, textures, clothing, touch, movement, lights)
a. Please specify

5. Does your child display any of the following maladaptive behaviors during mealtime?
a. Cries/tantrums
b. Tries to get out of seat
c. Leaves the table
d. Purposeful spit
e. Keeps mouth shut
f. Uses hands/arms to push food away
g. Eats too slow/fast
h. Chews but does not swallow
i. Holds food in his/her mouth/refuses to chew
j. Gags/coughs
k. Throws food
1. Other (please specify) 
6. What does your child do when a non-preferred food is offered?

7. Do you use any strategies to help your child eat? (i.e., positive reinforcement, behavioral reinforcement, behavioral techniques, reward system)

8. Please describe other concerns about your child's eating behavior or emotional condition?

\section{Parental Stress Section}

1. Do you enjoy mealtimes with your child?

Explain

2. Do you have a co-parent or is there another caregiver involved?

3. When I have problems/difficulties with the care of my child, I seek help or advice
a. True
b. False
c. Who? Explain

4. What do you do when your child displays maladaptive behaviors?

5. Have you noticed increased stress during mealtimes?

6. Which of the following methods do you use to reduce stress?
a. Listen to music
b. Watch tv/movie
c. Read
d. Write
e. Exercise
f. Take a nap
g. Get outdoors
h. Meditate
i. Clean
j. Talk with family/friends
k. Drink
1. Become aggressive
m. Eat
n. Cry
o. Go on a drive
p. Chew nails
q. Take it out on family
r. Other (please specify)

7. Have you found these strategies helpful?

\section{Explain}

8. Are you able to implement feeding strategies learned during therapy in the home environment?

9. Do you face any challenges when implementing these feeding strategies? If so, what are they?

10. Do you feel overwhelmed in the parenting role?

\section{Explain}

11. Do you find it difficult to balance the parenting role with other responsibilities? (i.e., having enough time for yourself) 
Explain

12. What is your daily stress level from 1-10?

$$
\begin{array}{llllllllll}
1 & 2 & 3 & 4 & 5 & 6 & 7 & 8 & 9 & 10
\end{array}
$$

13. What is your stress level during mealtime from 1-10?

$\begin{array}{llllllllll}1 & 2 & 3 & 4 & 5 & 6 & 7 & 8 & 9 & 10\end{array}$

14. Occupational therapists have the knowledge and expertise to assist you in creating a mealtime routine, if you could receive more support to help manage maladaptive behaviors and stress during mealtime routines which do you think would be more beneficial? (you may select more than one)

a. A brochure with stress management techniques

b. Parent-training course

c. Group support

d. Other; explain

Thank you for your willingness to participate in this study.

THIS PROJECT HAS BEEN REVIEWED AND APPROVED BY THE UNIVERSITY OF ST. AUGUSTINE FOR HEALTH SCIENCES INSTITUTIONAL REVIEW BOARD FOR THE PROTECTION OF HUMAN SUBJECTS.

IF YOU HAVE QUESTIONS OR CONCERNS, THOSE QUESTIONS OR CONCERNS SHOULD BE DIRECTED TO THE INSTITUTIONAL IRB CHAIR, DR. ELIZABETH ARDOLINO, EMAIL: EARDOLINO@USA.EDU, PHONE: 737-202-3343 


\section{APPENDIX D: MEMORANDUM OF UNDERSTANDING}

Course Number: OCT 6731 \& OCT 6832

Site Name: Hospital

Site Supervisor: Ashley K. Lee MOTR/L

Address: 2815 South Seacrest Blvd. Boynton Beach, FL 33435

Email address:

Phone: (561)-737-7733

Student Name: Andrea Patino

2021- April 2021

Student ID \#: 120845

Phone: (305)-984-7215

Mentor: Jennifer Sherm
Dates of experience requested: January

Email address: $\underline{\text { A.patino@usa.edu }}$

Doctoral Coordinator: Dr. Kasyan-Howe

Topic: Qualitative Exploration of Mealtime Routines, Behaviors, and Parental Stress of Children with Pediatric Feeding Disorders: Analysis of Occupational Performance

\section{Objectives:}

\section{Learning Objectives:}

- Compare and contrast the current multidisciplinary interventions that are being implemented for children with feeding disorders

- Develop broader understanding of the application of interventions in the home environment

- Compare and contrast the literature to parental responses from the interview questionnaire 
- Evaluate the family characteristics (socioeconomic status, child's gender, number of children) that may impact frequency of intervention implementation

- Describe advantages and disadvantages of current strategies used by parents to manage family dynamics, decrease stress, and increase mealtime participation

- Generalize themes from interviews and questionnaires that will help guide interventions

\section{Outcome Objectives:}

- Identify specific stressors parents experience during mealtime routines

- Identify stress reduction techniques to improve parent-child interactions

- Identify most frequently occurring maladaptive behaviors and the strategies used by parents to manage these behaviors

- Identify the similarities and differences of strategies used by families

Progress/Expected Completion Dates: Expected completion dates estimated for specific activities and for entire experience. Example: Weeks/Hours per week/dates

\begin{tabular}{|l|l|}
\hline Objectives & Progress/Expected Completion \\
\hline $\begin{array}{l}\text { Identify specific stressors parents experience } \\
\text { during mealtime routines }\end{array}$ & Jan-April 2021 \\
\hline $\begin{array}{l}\text { Identify stress reduction techniques to } \\
\text { improve parent-child interactions }\end{array}$ & Jan-April 2021 \\
\hline $\begin{array}{l}\text { Identify most frequently occurring } \\
\text { maladaptive behaviors and the strategies used } \\
\text { by parents to manage these behaviors }\end{array}$ & Jan-April 2021 \\
\hline $\begin{array}{l}\text { Identify the similarities and differences of } \\
\text { strategies used by families }\end{array}$ & Jan- April 2021 \\
\hline
\end{tabular}

\section{Plans for Supervision:}

Supervision and mentoring will be provided on an individualized basis and dependent on the needs of the student and demands of capstone. The supervisory relationship will demonstrate a mutual understanding of expectations through continuous monitoring of set objectives. Additional plans for supervision developed between student and site supervisor (as needed) Responsibilities of Student:

Student will participate in 448-560 hours within the mentored practice setting 
Student initiate and track progress towards identified objectives

Student will regularly communicate progress to all involved parties

Student will seek regular feedback on performance

\section{Responsibilities of Doctoral Coordinator:}

Ensures the consistency and quality of the capstone project and experience

Responsible for guiding student through entirety of the curriculum in preparation and completion of capstone

Advice and assists with securing capstone experience sites

Ensure student has met site requirements

Responsibilities of Site Supervisor:

Advise and assist regarding orientation, scheduling, resources, and requirements of site

Provide feedback on student performance as needed

Confirm student hours of participation

Responsibilities of Mentor:

Effectively communicate their knowledge expertise through mutual understanding of expectations

Provide feedback on proposal and paper

Attend poster presentation, if able

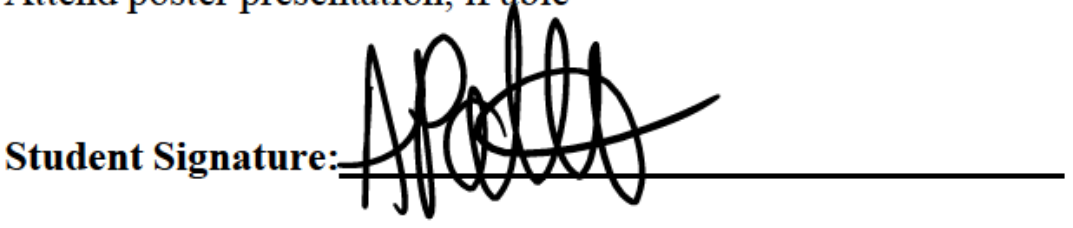

Date: $10 / 13 / 2020$

Doctoral Coordinator Signature:

Date:

Site Supervisor Signature: $\frac{\text { taun }}{y}$ K.feer Date: 